\title{
Phase Separation Kinetics in Unentangled Polymer Solutions under High-Rate Extension
}

\author{
Alexander N. Semenov ${ }^{1}$, Andrey V. Subbotin ${ }^{2,1}$ \\ ${ }^{1}$ Institut Charles Sadron, CNRS - UPR 22, Université de Strasbourg, \\ 23 rue du Loess, 67034 Strasbourg Cedex 2, France \\ ${ }^{2}$ A.V.Topchiev Institute of Petrochemical Synthesis, Russian Academy of Sciences, \\ Leninskii prosp. 29, Moscow, 119991, Russia
}

(December 20, 2016)

\begin{abstract}
Phase separation processes following high-rate extension in unentangled polymer solutions are studied theoretically. The flow-induced demixing is associated with the coil-stretch transition predicted in high-molecularweight polymer solutions at high enough Weissenberg numbers. The developed mean-field theory is valid in the dilute/semidilute solution regime where the stretched coils overlap strongly. We elucidate and discuss the main kinetic stages of the polymer/solvent separation process including: (i) growth of concentration fluctuations and formation of oriented protofibrils by anisotropic spinodal decomposition; (ii) development of well-defined highly-oriented and stiff fibrils forming an anisotropic network (cross-linked fiber); (iii) microphase separation and lateral collapse of the network yielding dense oriented fiber. These novel predictions are in qualitative agreement with experimental data.
\end{abstract}




\section{Introduction}

Flow can affect the polymer solution structure in a diverse number of ways. ${ }^{1}$ The main flow effects in solutions include anisotropic enhancement of concentration fluctuations leading to anisotropic scattering pattern, ${ }^{2-6}$ the flow-induced turbidity and phase separation, ${ }^{1,7-12}$ and shear banding. ${ }^{13-15}$

Several theoretical approaches have been devised to explain the flow-induced demixing phenomena. Within a phenomenological quasi-thermodynamic approach ${ }^{11}$ the flow effect is modelled by an additional free energy term which is linear in stress. Although this theory explains well the observed effects of flow on the cloud-point in polymer solutions, the approach is generally missing a solid physical justification. ${ }^{1}$ Another theory ${ }^{16}$ explains the shear-demixing effect in entangled semidilute polymer solutions by concentration dependence of $N_{e}$, the number of units per entanglement. A more realistic theory of flow-induced demixing effects is hinged on a coupling between polymer elastic stress and polymer concentration. ${ }^{3-6,1}$ An increase of tensile stress with concentration creates a stress gradient directed towards a more concentrated region thereby driving more polymer into such regions. The idea of the stress-concentration coupling (SCC) allowed to explain a number of flow-induced phenomena in entangled polymer solutions including amplification of concentration fluctuations ${ }^{3-6}$ and shear banding. ${ }^{15}$

Most theories mentioned above seem to be applicable to entangled polymer solutions. However, it appears that even the apparently simpler unentangled regime is not yet fully understood. For example, the SCC theory does not predict any phase separation in the presence of elongational flow in this regime. ${ }^{6}$ By contrast, recent rheological data show that such phase separation (extensioninduced solvent release) is possible for a number of polymer solutions. ${ }^{17,19,20}$ It is noteworthy that the SCC approach, when applied to unentangled systems at moderate Weissenberg numbers ( $\mathrm{Wi} \sim 1$ ), predicts only a weak driving force for concentration wave amplification which (being comparable, albeit opposite, to the ideal-gas osmotic pressure effect) is normally much weaker than the effect of excluded-volume interactions of polymer segments. The reason is that polymer coils are moderately deformed for $\mathrm{Wi} \sim 1$, so the flow-induced chain tension is $\sim T / R_{\text {coil }}$, and its contribution to stress is only $\sim T$ per chain (where $T$ is temperature in energy units, and $R_{\text {coil }}$ is the coil size).

Most of experimental evidence on phase separation in polymer solutions under extension concerns unentangled or moderately entangled systems. ${ }^{18,17,19,20}$ The underlying rheological phenomena are complicated and are not understood well theoretically, hence we chose to start their analysis with a simpler unentangled regime. Recently we considered the demixing instability induced by a sufficiently fast elongational flow in unentangled polymer solutions. ${ }^{21}$ The instability is associated with the extension-induced coil to stretched coil transition. The driving force for the demixing effect is related to a decrease of the osmotic pressure due to alignment of polymer segments in a stretched chain. The magnitude of this thermodynamic driving force is proportional to $\phi N T$ per polymer chain, where $\phi$ is polymer volume fraction, so it dominates the SCC effect as long as $\phi N T \gg T$. It is predicted $^{21}$ that uniaxial extensional flow can induce demixing in polymer solutions if the extension rate exceeds some critical value $\left(\mathrm{Wi}>\mathrm{Wi}_{c} \sim 1\right)$. This effect differs from the capillary breakage of polymer jets due to Plateau-Rayleigh 
instability $^{22,23}$ which does not necessarily imply any demixing of the system.

The aim of the present study is to describe theoretically how the phase separation process in an unentangled (dilute or semidilute) polymer solution proceeds upon its fast extension, and what sort of eventual polymer jet structure is expected. ${ }^{24}$ Note that the demixing mechanism considered here is akin to, but is different from the conformational deformation effect of shear flow leading to a shift of the critical point for polymer solutions. ${ }^{26}$

The theoretical approach of ref. 21 is described in the next section. The dynamics of phase separation (spinodal decomposition of the solution) is considered in the 3rd section, which is followed by a Discussion of the main results.

\section{The model and the coil-stretch transition}

Let us consider a rather dilute solution of semiflexible polymer with contour length $L=N l_{1}$ and mean concentration of repeat units $c(N \gg 1$ is the polymerization index, $l_{1}$ is length per repeat unit). The crossover concentration $c^{*} \sim N / R_{\text {coil }}^{3}$, where $R_{\text {coil }}$ is the equilibrium coil size, corresponds to nearly overlapping coils.

The study is focussed on the dilute/semidilute transition region $c \sim c^{*}$ assuming that $\vartheta \sim c / c^{*}$ is not too low and not too large, so that the coils interact, but the system is not entangled (see point 3 of section 4 and the Appendix for more detailed criteria). For simplicity we also assume the marginal solvent regime ${ }^{28,29}$ with nearly Gaussian coils, $R_{\text {coil }} \sim R_{0} \sim a_{s} N^{1 / 2} \quad\left(a_{s}\right.$ is the polymer statistical segment length). ${ }^{27}$

Now suppose that homogeneous uniaxial elongational flow field is generated in the system, with flow velocity

$$
v_{z}=\dot{\epsilon} z, \quad v_{x}=-\dot{\epsilon} x / 2, \quad v_{y}=-\dot{\epsilon} y / 2
$$

where $\dot{\epsilon}$ is extension rate. De Gennes ${ }^{31,32}$ showed that if $\dot{\epsilon}$ exceeds some critical rate, the polymer coils in a dilute solution $\left(c<c^{*}\right)$ become strongly deformed and stretched along the main flow axis ( $z$-axis). This is a discontinuous transition with a significant increase of coil size from $R_{\text {coil }} \sim R_{0} \propto N^{0.5}$ to $R_{z} \sim N$ (Figure 1a).

The nature of this transition can be clarified in the following way. It is the hydrodynamic friction force $\Delta H$ that pulls the two parts of a chain away from each other (Figure 1b). For moderately stretched chains $\left(s=R_{z} / L \ll 1\right)$ the friction force increases quadratically with $s$ (cf. eq. (1)), while the elastic force $H_{e l}$ which opposes the stretching increases slower $\left(H_{e l} \propto s\right.$ for Gaussian coils), so an abrupt stretching transition occurs as a result. Note that a strong increase of the coil longitudinal size $R_{z}$ is accompanied by its moderate lateral collapse. However, the lateral size $R_{x}$ remains nearly Gaussian, $R_{x} \sim a_{s} N^{1 / 2}$ unless $s$ is very close to 1 (corresponding to full chain extension).

Recently ${ }^{21}$ we analyzed this transition quantitatively using the simple and realistic wormlike (or persistent chain) model of polymer chains. ${ }^{33}$ Consider a chain whose midpoint is located near the origin, where flow velocity vanishes (see Figure 1b), so the chain does not move. The friction force (in $z$-direction) on a polymer fragment of length $\Delta z$ is

$$
\Delta H \simeq \zeta_{\|} v_{z} \Delta z
$$


where $\zeta_{\|} \simeq 2 \pi \eta_{s} / k_{H}$ is longitudinal friction constant (per unit length) ${ }^{35}, \eta_{s}$ is solvent viscosity, $k_{H} \simeq \ln \left(\xi_{H} / d\right)$ is the hydrodynamic factor, $d$ is the effective chain thickness, and $\xi_{H}$ is hydrodynamic screening length comparable to the typical lateral distance between stretched chains, $\xi_{H} \sim\left(\frac{N}{c L s}\right)^{1 / 2}=\left(c l_{1} s\right)^{-1 / 2}$. Thus

$$
k_{H} \simeq \frac{1}{2} \ln \left(\frac{1}{\phi s}\right)
$$

where $\phi=v_{1} c$ is polymer volume fraction $\left(v_{1} \equiv \frac{\pi}{4} l_{1} d^{2}\right.$ is the intrinsic volume per monomer unit). The $s$-dependence of $k_{H}$ can be neglected for $s \sim 1$ since $\phi \ll 1$. Eq. (2) is valid both in dilute $\left(c<c^{*}\right)$ and semidilute $\left(c>c^{*}\right)$ regimes, and it shows that the friction depends on concentration just weakly (logarithmically). Note that eq. (1) implies that the total friction force basically scales as $R_{z}^{2}$ since both $\Delta z \propto R_{z}$ and $v_{z} \propto R_{z}$.

The elastic force is defined by the elastic free energy per chain ${ }^{21}$ :

$$
F_{e l} \simeq \frac{T L}{2 l} \widetilde{F}(s)
$$

where $l$ is Kuhn segment length $\left(l=a_{s}^{2} / l_{1}\right)$, and the function $\tilde{F}(s)$ is parametrically defined by equations

$$
\widetilde{F}=A \operatorname{coth}(A)-1, \quad s=\operatorname{coth}(A)-1 / A
$$

The flow rate can be characterized by the reduced parameter

$$
U=2 \dot{\epsilon} \tau_{R} \equiv 2 \mathrm{Wi}
$$

where

$$
\tau_{R}=\frac{\pi \eta_{s} L^{2} l}{18 T k_{H}}
$$

is the typical relaxation time of the stretched chain (note that $\tau_{R} \propto N^{2}$, so it is called the Rouse time below). The equilibrium order parameter $s=s(U)$ (the stretching degree) can be obtained by minimization of the effective total free energy involving the elastic and friction contributions (cf. ref. 21). This operation boils down to

$$
\min _{s}\left(s A-U s^{2}\right)
$$

where $A=A(s)$ is obtained by solving the second eq. (3). It is shown ${ }^{21}$ that the stretched coil state is absolutely stable if $U>U_{c} \approx 6.6$. In this regime the degree of stretching is significant, $s>s_{c}=0.64$, and it increases further with extension rate.

The polymer osmotic pressure depends on the orientation state of polymer segments, and so does the free energy of their interactions $F_{\text {int }}$. In the isotropic state (for $\phi \ll d / l$ ) the interactional free energy (per unit volume) can be written in the mean-field approximation as

$$
F_{i n t} / T \simeq \frac{v}{2} c^{2}+O\left(c^{3}\right)
$$


The second virial coefficient $v$ of polymer segment interactions results from a competition between their steric repulsion $\left(v_{s}\right)$ and dispersion attraction $\left(-v_{a}\right)$ : $v=v_{s}-v_{a}$. Within the standard van-der-Waals approximation $v_{s}$ is independent of $T$ and $v_{a} \propto 1 / T$, so $v=v_{s}(1-\Theta / T)$, where $\Theta$ is the theta-temperature. It is assumed that $T$ is slightly above $\Theta$, so $k_{T} \equiv 1-\Theta / T \ll 1$ (say, $k_{T} \lesssim 0.1$ ). This condition is consistent with the marginal solvent regime assumed above, justifying the mean-field approach adopted here.

Let's consider the system after a fast extension with $U>U_{c}$. In this case the chain segments are oriented, and $F_{\text {int }}$ depends on the order parameter $s$. The free energy of interactions of overlapping wormlike chains obtained using the mean-field theory ${ }^{33,21}$ is:

$$
F_{\text {int }} / T=0.5 c^{2} v_{s}(I(s)|\ln (1-\phi)| / \phi-\Theta / T)
$$

where $v_{s}=(\pi / 2) d l_{1}^{2}$,

$$
I(s)=\frac{4}{\pi}\langle\sin \beta\rangle
$$

and $\beta$ is the angle between two randomly chosen polymer segments. The function $I(s)$ accounts for anisotropy of steric interactions; it is calculated in ref. 21 ; it decreases with $s$ as $I(s) \sim \sqrt{1-s}$ for $1-s \ll 1 .^{34}$ The anisotropy of attractive monomer interactions is neglected in eq. (6) (cf. the second term in brackets) as these interactions are typically more long-range and therefore more isotropic than the steric interactions.

The polymer osmotic pressure is

$$
\Pi=\Pi_{i d}+\Pi_{i n t}
$$

where $\Pi_{i d}=c T / N$ is the ideal-gas pressure, $\Pi_{i n t}$ is the pressure contribution due to interactions of polymer segments, $\prod_{\text {int }}=-F_{\text {int }}+c \partial F_{\text {int }} / \partial c \simeq T v^{*} c^{2} / 2$ for $\phi \ll 1$. Here

$$
v^{*}=v_{s}\left(I(s)-\frac{\Theta}{T}\right)
$$

is the total virial coefficient. The virial parameter $v^{*}$ results from the balance of monomer repulsion (the first term $I(s)$ in brackets) and attraction (the second term $\Theta / T)$. The reduced osmotic modulus is

$$
\kappa=\frac{1}{T} \frac{\partial \Pi}{\partial c} \simeq \frac{1}{N}+v^{*} c
$$

Upon coil stretching the repulsion decreases (since $I(s)$ decreases with the order parameter $s$ ), so both $v^{*}$ and the osmotic modulus $\kappa$ can change their sign becoming negative in the stretched state. This always happens for sufficiently strong stretching (large $s$ ) provided that

$$
c\left|v^{*}\right| N=2 \phi \frac{L}{d}\left|k_{T}\right| \gg 1
$$

where $k_{T} \equiv I(s)-\frac{\Theta}{T}$. The ideal-gas contribution is negligible in this regime. The above condition is assumed in what follows. It requires that $\phi L / d \gg 1$. In terms 
of $\vartheta \sim c / c^{*}$ it means that $\vartheta \gg N^{-1 / 2}$ (since $\phi^{*}=v_{1} c^{*} \sim 1 / \sqrt{N}$ ), so it allows for both dilute and semidilute regimes. Note that the condition (10) also ensures that the SCC effect is negligible since its magnitude (for $\mathrm{Wi}=\dot{\epsilon} \tau_{R} \sim 1$ ) is comparable with that of the ideal-gas contribution.

It is important to note that the coil volume increases dramatically upon stretching: In fact, the longitudinal coil size increases by a factor of $\sim \sqrt{N}$, while the lateral size remains nearly the same $\left(\sim R_{0}\right)$. Therefore, the initially dilute system can enter the semidilute regime of strongly overlapping chains upon stretching if the condition (10) is satisfied. That is why the mean-field theory of osmotic effects considered above is valid for stretched chains also in the formally dilute regime, $1 / \sqrt{N} \ll \vartheta \ll 1$.

\section{Polymer/solvent demixing}

In the previous section we recalled that polymer chains can be significantly stretched if the elongation rate $\dot{\epsilon}$ is fast enough: $\mathrm{Wi}=\dot{\epsilon} \tau_{R}>\mathrm{Wi}_{\boldsymbol{c}} \approx 3.3$. It was also shown that the osmotic modulus $\kappa$ can become negative upon chain stretching. In this case a homogeneous system of stretched chains is rendered unstable with respect to polymer/solvent segregation. The demixing process implies that certain concentration fluctuation modes grow in time (the spinodal decomposition). The early stage of this process is considered below following the classical Cahn-Hilliard approach.

Here we choose to focus on the simplest case of instantaneous stretching (at $t=0$ ) from isotropic state to $s>s^{*}$, followed by the flow cessation, where $s^{*}$ is defined by the condition $I\left(s^{*}\right)=\Theta / T$. A continuous stretching scenario is considered in section 4. Two basic possibilities can be distinguished regarding the flow at $t>0$ : (i) no flow, $\dot{\epsilon}=0$, and (ii) the extension rate $\dot{\epsilon}=$ const $\sim 1 / \tau_{R}$ is such as to keep the degree of stretching unchanged, $s=$ const. In the first case the stretched coils tend to retract, so that $s=s(t)$ decreases with time. However, this process is slow (its timescale is $\tau_{R}$ ) and can be neglected since, as shown below, the characteristic time of spinodal decomposition, $t_{l}$, is much shorter than $\tau_{R}$. For the same reason the polymer advection can be neglected in the second case.

\subsection{Spinodal decomposition}

To study the kinetics of spinodal decomposition upon fast uniaxial extension along $z$-axis we employ the mean-field quasi-thermodynamic approach tracing back to the classical Cahn-Hilliard theory ${ }^{58,59}$. The macroscopic state of the system is defined in terms of the concentration field $c(\underline{r})$. The thermodynamic potential (free energy) of the system $\mathcal{F}$ is considered as a functional of $c(\underline{r})$. The generalized chemical potential $\mu$ is then defined as the variational derivative of $\mathcal{F}$ :

$$
\mu(\underline{r})=\frac{\delta \mathcal{F}}{\delta c(\underline{r})}
$$

The continuity equation is $\frac{\partial c}{\partial t}=-\nabla \underline{J}$, where the current $\underline{J}$ is proportional to the local force $\nabla \mu$ : 


$$
\underline{J}=\underline{v} c-\left(c / \zeta_{1}\right) \nabla \mu
$$

where $\underline{v}$ is the flow velocity (neglected below) and $\zeta_{1}$ is the monomer friction constant. The kinetic equation can be then written as

$$
\frac{\partial c}{\partial t}=-\nabla \underline{J}=\nabla\left(\frac{c}{\zeta_{1}} \nabla \mu\right)
$$

We are mostly interested in the lateral chain motion (perpendicular to the extension axis $z$, cf. Figure 1b). The corresponding friction constant per chain is $N \zeta_{1} \simeq$ $4 \pi \eta_{s} R_{z} / k_{H}$ (cf. ref. 35 ), so

$$
\zeta_{1} \simeq 4 \pi \eta_{s} l_{1} s / k_{H}
$$

The free energy $\mathcal{F}$ includes two contributions:

$$
\mathcal{F}_{\text {int }} \simeq \frac{T}{2} v^{*} \int c(\underline{r})^{2} \mathrm{~d}^{3} r
$$

due to interactions of polymer segments (the second virial term only is kept here since concentration is low, $\phi_{0} \lesssim 1 / \sqrt{N} \ll 1$ ), and the conformational free energy $\mathcal{F}_{\text {conf }}$. For small fluctuations, $|\delta c| \ll c_{0}$, the latter can be related to the chain formfactor $S_{0}(q)$ for the system of non-interacting (but stretched) chains using the RPA approach (cf. ref. 32):

$$
\mathcal{F}_{\text {conf }} \simeq \frac{T}{2 c_{0}} \int \delta c(\underline{r}) K_{0}\left(\underline{r}-\underline{r}^{\prime}\right) \delta c\left(\underline{r}^{\prime}\right) \mathrm{d}^{3} r \mathrm{~d}^{3} r^{\prime}
$$

where $c_{0}$ is the mean concentration $\left(\phi_{0}=v_{1} c_{0}\right), K_{0}$ is the kernel function whose Fourier transform is $1 / S_{0}(q)$, and

$$
S_{0}(q)=\frac{1}{N} \sum_{i j}\left\langle e^{\mathrm{i} \underline{\underline{i}} \cdot\left(\underline{r}_{i}-\underline{r}_{j}\right)}\right\rangle
$$

Here $i, j$ run over all units of one ideal stretched chain, and $r_{i}$ is the position of the $i$-th unit of the chain. Thus, for $|\delta c| \ll c_{0}$ the free energy $\mathcal{F}=\mathcal{F}_{\text {int }}+\mathcal{F}_{\text {conf }}$ can be written as

$$
\mathcal{F} \simeq \frac{T}{2 V} \sum_{q \neq 0}\left(v^{*}+\frac{1}{c_{0} S_{0}(q)}\right)\left|c_{q}\right|^{2}
$$

where $c_{q}$ is the Fourier transform of $\delta c(\underline{r}), V$ is the total volume. ${ }^{36}$

To calculate $S_{0}(q)$ we note that the lateral statistics of the stretched chain remain Gaussian at the relevant length-scale $\lambda \gg l$ : in particular, the mean-square $x$-projection of the end-to-end distance $R_{x}$ is $\left\langle R_{x}^{2}\right\rangle=2 a^{2} N$, where $a=a(s)$. For persistent chains the function $a(s)$ can be approximated as $a(s) \approx\left(1-s^{2}\right) a_{s} / \sqrt{6}$. Thus, we get using eq. (13) for $l \ll 1 / q \ll R_{0}$ :

$$
S_{\mathbf{0}}(q) \simeq 2 \int_{\mathbf{0}}^{\infty} \mathrm{d} n e^{-q^{2} a^{2} n} \cos \left(q_{z} s l_{1} n\right)=\frac{2 q^{2} a^{2}}{\left(q^{2} a^{2}\right)^{2}+\left(q_{z} s l_{1}\right)^{2}}
$$

The above equation is valid if $\left|q_{z}\right| \ll q$. This regime is of the main interest as verified later. 
Using eqs. (15), (14) the linearized kinetic equation (11) can be written as ${ }^{37}$

$$
\frac{\mathrm{d} c_{\underline{q}}}{\mathrm{~d} t} \simeq \Gamma(\underline{q}) c_{\underline{q}}
$$

where the growth rate

$$
\Gamma(\underline{q})=-\frac{T}{\zeta_{1}} q^{2}\left(c_{0} v^{*}+1 / S_{0}(q)\right) \simeq \frac{T}{\zeta_{1}} q^{2}\left(|\kappa|-\frac{q^{2} a^{2}}{2}-\frac{q_{z}^{2} s^{2} l_{1}^{2}}{2 q^{2} a^{2}}\right)
$$

Here $\kappa \simeq c_{0} v^{*}<0$, and as before we assume $1 \gg|\kappa| \gg 1 / N$. The maximum growth rate $\Gamma^{*} \simeq \frac{T}{2 \zeta_{1} a^{2}}|\kappa|^{2}$ corresponds to $q^{2}=|\kappa| / a^{2}$ and $q_{z}=0$. The critical wave vector $q^{*}=\sqrt{|\kappa|} / a$ is therefore oriented perpendicular to the $z$-axis. The corresponding length scale

$$
\xi=2 \pi / q^{*}=2 \pi a / \sqrt{|\kappa|} \sim\left(\frac{l d}{\phi_{0}\left|k_{T}\right|}\right)^{1 / 2}
$$

gives the typical lateral spacing of emerging domains (regions of elevated concentration, $c>c_{0}$ ). We can estimate the number of chains per domain: $n_{f} \sim \xi^{2} c_{0} l_{1} s$, where $c_{0} l_{1} s$ is 2-dimensional concentration of chains (number of chains intersecting an xy plane per unit area). Hence

$$
n_{f} \sim \frac{4 \pi^{2} a^{2}}{c_{0}\left|v^{*}\right|} c_{0} l_{1} s \approx \frac{l}{d} \frac{4 s\left(1-s^{2}\right)^{2}}{\left|k_{T}\right|}
$$

where $k_{T}=I(s)-\Theta / T$. We consider the regime $\left|k_{T}\right| \ll 1$, so $n_{f} \gg 1$.

Concentration fluctuations grow exponentially in time in the 'linear' regime, $|\delta c(\underline{r})| \ll c_{0}$ :

$$
c_{\underline{q}}(t) \simeq c_{\underline{q}}(0) e^{\Gamma(\underline{q}) t}
$$

The function $\Gamma(\underline{q})$ close to $\underline{q}^{*}$ is approximately

$$
\Gamma=\Gamma^{*}-\Delta \Gamma, \quad \Delta \Gamma / \Gamma^{*} \simeq\left(\frac{2 \Delta q}{q^{*}}\right)^{2}+\left(\frac{q_{z}}{q_{z}^{*}}\right)^{2}
$$

where $\Delta q=q-q^{*}$ and $q_{z}^{*}=|\kappa| /\left(s l_{1}\right)$ is the characteristic longitudinal wave-number. The fluctuation modes mostly contributing after a growth time $t$ are defined by the condition $t \Delta \Gamma \lesssim 1$. As shown below, $\Gamma^{*} t$ cannot be very large in the early regime (it is limited by a log-factor), hence the condition reduces to $\Delta \Gamma / \Gamma^{*} \lesssim 1$ leading to the conclusion that nearly all the fluctuation modes with $|\Delta q| \lesssim q^{*} / 2$ and $\left|q_{z}\right| \lesssim q_{z}^{*}$ contribute in the concentration pattern at the end of the early stage.

The time of this stage $t_{l}$ can be estimated by the condition $\left|\delta c\left(t_{l}\right)\right| \sim c_{0}$, where $|\delta c(t)|$ is the typical amplitude of concentration fluctuation in the relevant domain of lateral size $\xi$ and longitudinal size $\xi_{z} \sim 1 / q_{z}^{*}$. With the broad spectrum of growing modes revealed above, the fluctuation amplitude in real space approximately follows the exponential law $|\delta c(t)| \sim|\delta c(0)| e^{\Gamma^{*} t}$. An estimate of $|\delta c(0)|$ (smoothed over the relevant domain) is not a trivial task (involving also a consideration of the preliminary stretching stage). The result, however, is simple and natural: 


$$
|\delta c(0)| / c_{0} \sim 1 / \sqrt{n_{f}}
$$

Therefore we arrive at the condition $e^{\Gamma^{*} t_{l}} \sim \sqrt{n_{f}}$ defining $t_{l}$ :

$$
\Gamma^{*} t_{l} \sim 0.5 \ln n_{f}
$$

The r.h.s. of this relation, being formally large for $n_{f} \gg 1$, is not important in practice: $0.5 \ln n_{f} \sim 1$ for $n_{f} \lesssim 40$. Therefore, the duration of the early stage can be estimated neglecting the log-factor as:

$$
t_{l} \sim 1 / \Gamma^{*} \sim 6\left(1-s^{2}\right)^{2} s \tau_{R} /\left(\left|k_{T}\right| \phi_{0} L / d\right)^{2}
$$

so $t_{l} \ll \tau_{R}$ since $\left|k_{T}\right| \phi_{0} L / d \gg 1$ (cf. eq. (10)).

The breadth of the spectrum of growing modes $\left(\Delta q \sim q^{*}\right)$ means that the emerging domains are disordered and irregular in the xy plane also because of interference of modes with different $\underline{q}$-orientations (cf. Figure 2a). The important feature, however, is that the domains are strongly elongated along $z$-axis: the typical domain longitudinal size

$$
\xi_{z} \sim s l_{1} /|\kappa| \sim d /\left(\phi_{0}\left|k_{T}\right|\right)
$$

is much longer than its lateral size $\sim \xi / 2 \sim \pi a / \sqrt{|\kappa|}$ (this condition obviously implies also that $\left.\left|q_{z}\right| \ll q^{*}\right)$ :

$$
\xi_{z} / \xi \sim \frac{s \sqrt{3}}{\pi\left(1-s^{2}\right)}\left(\frac{l}{d} \phi_{0}\left|k_{T}\right|\right)^{-1 / 2} \gg 1
$$

since the system was assumed to be rather dilute $\phi_{0} l / d \ll 1$ (not to mention that $\left|k_{T}\right|$ is also small). It is also important that stretched polymer chains are still much longer than $\xi_{z}$ :

$$
R_{z} / \xi_{z} \sim N|\kappa|=2 \phi\left|k_{T}\right| L / d \gg 1
$$

This geometry of denser domains (Figure 2b) allows to consider them as precursors of fibrils (or protofibrils) whose formation is studied in the next section.

\subsection{Fibril formation}

During the early stage of phase separation $\left(|\delta c| / c_{0} \ll 1\right)$ considered in the previous section the polymer-depleted $(\delta c<0)$ and concentrated $(\delta c>0)$ regions have similar geometry, and the whole structure is isotropic in the xy plane (cf. Figure 2a). At the end of the early stage the concentration contrast between dense and dilute regions is significant, $|\delta c| / c_{0} \sim 1$, so most of the polymer is accumulated in the more concentrated regions (this notion is already taken into account in the estimate of the number of chains in a fibril cross-section, cf. eq. (18)). What happens later? The concentration in polymer-rich regions tends to increase further (since the equilibrium polymer concentration, $\phi_{c}$, is much higher than $\phi_{0}$, cf. eq. (22)). As the total amount of polymer is constant, this densification process necessarily leads to a decrease of volume fraction $\varphi$ of the polymer-rich domains, so $\varphi$ becomes small. The polymer domains then become the minor phase by volume, 
and they appear as separate (polydisperse) disks in the xy cross-section (Figure 3a). On the other hand, the domains stay elongated in the third $(z)$ dimension, so they can be viewed as fibrils. It is the lateral attraction of oriented polymer chains that drives their aggregation into well-defined cylindrical filaments (Figure 3b). Note that the number of chains per fibril, $n_{f}$ (cf. eq. (18)) does not depend either on concentration or on polymer molecular weight: $n_{f} \sim(l / d) /\left|k_{T}\right|$ depends mainly on $T$ and the chain stiffness parameter $l / d$. For the PAN solutions ${ }^{17,18}$ we expect $n_{f} \gtrsim 30$ since $\left|k_{T}\right| \lesssim 0.1$ and $l / d \sim 5$ (cf. the Appendix).

Let us turn to the global structure of the system of fibrils illustrated in Figure 3b. It shows a network of fibrils with no free ends. Why the network? In fact, the branched structure is necessarily formed already during the transition from the early stage (amplification of concentration fluctuations) to the stage of fibril development. The domain (fibril) branching stems from the randomness of the growing fluctuation modes during the early stage: two polymer chains belonging to the same fibril at $z_{1}$ may belong to different (neighboring) fibrils at $z_{2}$ (see Figure 4) if $\left|z_{2}-z_{1}\right| \gtrsim \xi_{z}$. This argument shows that the typical fibril strand length (between the neighboring junctions) is $\sim \xi_{z}$.

Now, why no free ends? A fibril termination is virtually impossible as it consists of many polymer chains whose ends are not really correlated. An existence of a fibril end implies all the constituent polymer chains have ends with nearly the same $z$-coordinates (within an error $\Delta z \sim \xi_{z}$ ). The probability $p$ of this event is extremely low:

$$
p \sim\left(\xi_{z} / R_{z}\right)^{n_{f}} \sim\left(\frac{L}{d} \phi_{0}\left|k_{T}\right|\right)^{-n_{f}}
$$

Obviously, $p \ll 1$ since $\frac{L}{d} \phi_{0}\left|k_{T}\right| \gg 1$ and $n_{f} \gg 1$.

Let us return to the fibril densification process. Right after the early stage of spinodal decomposition the polymer concentration inside a fresh fibril is $\phi_{f} \sim \phi_{0}$. The equilibrium concentration in the polymer phase, $\phi_{c}$, is much higher ${ }^{21}$ :

$$
\phi_{c} \simeq 1-I(s) T / \Theta=(T / \Theta)\left|k_{T}\right|
$$

This concentration is defined by the condition $\Pi=0$, where $\Pi$ is polymer osmotic pressure,

$$
\Pi \simeq \Pi_{i n t}=-F_{\text {int }}+c \partial F_{i n t} / \partial c
$$

(The ideal-gas contribution $\Pi_{i d}=c T / N$ is negligible as the chains are very long, $(L / d) \phi\left|k_{T}\right| \gg 1$.) As a very rough estimate, $\phi_{0} \propto 1 / \sqrt{N}$, while $\phi_{c}$ is independent of $N$, so $\phi_{c} \gg \phi_{0}$ for long chains (for example, $\phi_{0} \sim 1 \%$ while $\phi_{c} \sim\left|k_{T}\right| \sim 0.1$ for $s \lesssim 0.8)$. Hence, there is a strong thermodynamic force for fibril compaction. The osmotic energy gain per monomer entering the dense phase (the fibril) is

$$
\Delta f_{i n t} \sim T \phi_{c} \frac{l_{1}}{d}\left|k_{T}\right|
$$

The fibrils cannot contract longitudinally as they are highly stretched and incorporated in the network structure. Therefore, their diameter $d_{f}$ should decrease. This process is analyzed below. 
A fibril of diameter $d_{f}$ is shown in Figure 5; initially $d_{f} \sim \xi / 2$. The fibril lateral collapse is governed by a balance between the polymer attraction energy gain and viscous dissipation due to polymer/solvent friction:

$$
\mathrm{d} \mathcal{F}=-\mathcal{D} \mathrm{d} t
$$

where $\mathcal{F} \approx \mathcal{F}_{\text {int }}$ is the fibril free energy per unit length, and $\mathcal{D}$ is the corresponding dissipation rate. The free energy $\mathcal{F}_{\text {int }}=A F_{\text {int }}$, where $A=(\pi / 4) d_{f}^{2}$ is the fibril crosssection area, and $F_{\text {int }}$ is defined in eq. (6) with $\phi=\phi_{f}=\frac{n_{f} d^{2}}{s d_{f}^{2}}$. The dissipation rate $\mathcal{D}=A \zeta_{1} \frac{\phi_{f}}{v_{1}}\left\langle v^{2}\right\rangle$, where $v=v(r)$ is the radial velocity of polymer segments, $r$ is the distance to the fibril axis, $\left\langle v^{2}\right\rangle=\frac{1}{A} \int_{0}^{d_{f} / 2} 2 \pi r v^{2} \mathrm{~d} r$. We assume uniform collapse: $v=r \mathrm{~d} \ln d_{f} / \mathrm{d} t$, so $\left\langle v^{2}\right\rangle=\frac{1}{32} \frac{d_{f}^{2}}{\phi_{f}^{2}} \dot{\phi}_{f}^{2}$, where $\dot{\phi}_{f}=\frac{\mathrm{d} \phi_{f}}{\mathrm{~d} t}$. Eq. (24) can be then transformed as

$$
\frac{K}{\phi_{f}^{3}} \dot{\phi}_{f}=\left(\frac{\Theta}{T}-\frac{I(s)}{1-\phi_{f}}\right)
$$

where $K=\frac{\zeta_{1} n_{f} d^{3}}{32 T s l_{1}}$. In the region $\phi \ll \phi_{c}$ the solution (with initial condition $\left.\phi_{f}(0)=\phi_{0}\right)$ is

$$
\phi_{f}=\phi_{0} / \sqrt{1-t / t_{c}}
$$

where $t_{c}=\frac{K}{2\left|k_{T}\right| \phi_{f}(0)^{2}}$ is the time of the compaction stage. Eq. (25) cannot be applied at the end of this stage, at $1-t / t_{c} \lesssim\left(\phi_{0} / \phi_{c}\right)^{2} \ll 1$, but this region gives only a minor correction to the whole time $t_{c}$. Comparing $t_{c}$ with eq. (20) for $t_{l}$ we find

$$
t_{c} \sim t_{l}
$$

that is, the early growth stage and the fibril compaction stage are characterized by essentially the same time.

It is worth noting that the fibril surface energy contribution was neglected in the above treatment. This contribution is never dominant, so it does not cause any significant change of the results. The surface energy per unit length $P \approx \pi d_{f} \gamma_{f}$ ( $\gamma_{f}$ is the surface tension) is of the order of the osmotic energy gained on fibril lateral compaction $P \sim \Delta f_{i n t} n_{f} / l_{1} \sim T l \phi_{c} / d^{2}$ (cf. eq. (23)). It is interesting that $P$ can be also considered as the thermodynamic driving force tending to shorten a fibril. In should be compared with the total elastic tension force due to all stretched chains,

$$
F_{t} \sim n_{f} T / l
$$

where the chain tension is $\sim T / l$ for $s \sim 0.7$. This leads to $P / F_{t} \sim l \Delta f_{\text {int }} /\left(l_{1} T\right) \sim$ $\left|k_{T}\right|^{2} l / d$. Thus, although $P / F_{t}$ can be large for very stiff polymers, it is expected to be small for not too rigid polymers like $\mathrm{PAN}$ : in this case $P / F_{t} \sim\left|k_{T}\right|^{2} l / d \lesssim 0.1$ (cf. the Appendix), so the surface energy contribution to fibril tension can be neglected.

To summarize, a highly anisotropic network of well-defined oriented fibrils is formed at the end of the second decomposition stage (lateral compaction of polymer 
domains). The eventual volume concentration of polymer inside fibril is $\phi_{c} \sim\left|k_{T}\right|$ (cf. eq. (22)), so the fibril density strongly increases during this stage: $\phi_{c} / \phi_{0} \gg 1$. The initial fibril diameter, $d_{f}(0) \sim \xi / 2 \sim \sqrt{l d /\left(\phi_{0}\left|k_{T}\right|\right)}$ (cf. eq. (17)), is much shorter than the reference coil size $R_{0} \sim \sqrt{L l}: \quad d_{f}(0) / R_{0} \sim\left(\phi_{0}\left|k_{T}\right| L / d\right)^{-1 / 2} \ll 1$. The final diameter of mature fibril is

$$
d_{f} \sim d_{f}(0) \sqrt{\phi_{0} / \phi_{c}} \sim \sqrt{l d} /\left|k_{T}\right|
$$

it is much smaller than $d_{f}(0)$. The number of chains per fibril is $n_{f} \sim(l / d) /\left|k_{T}\right|$ (cf. eq. (18)). If the fibril remains liquid, its rigidity (Kuhn) segment is

$$
l_{f} \simeq n_{f} l \sim l^{2} /\left(d\left|k_{T}\right|\right)
$$

The fibril strand length (between the neighboring network junctions) is $L_{f} \sim \xi_{z} \sim$ $d /\left(\phi_{0}\left|k_{T}\right|\right)$. Hence $L_{f} / l_{f} \sim d^{2} /\left(l^{2} \phi_{0}\right)$, i.e., typically the strands are semiflexible, $L_{f} \gg l_{f}$, if the initial solution was dilute enough, $\phi_{0} \ll(d / l)^{2}$. The fibrils themselves occupy a small fraction $\varphi$ of the total volume, $\varphi \approx \phi_{0} / \phi_{c} \sim \phi_{0} /\left|k_{T}\right| \ll 1$.

Note that an exchange of chains between fibrils is suppressed during the segregation process we consider: For a condensed chain fragment of length $\Delta L_{z}$ to escape from its fibril, it needs to get an energy $E_{e s} \sim \phi_{c}\left|k_{T}\right| \Delta L_{z} / d$. This energy is high not only for the whole chain, but also for the minimal fragment $\Delta L_{z} \sim \xi_{z}$ : in this case $E_{e s} / T \sim \frac{\phi_{c}}{\phi_{0}}$, so $E_{e s} \gg T$ since $\phi_{0} \ll \phi_{c}$. Hence, polymer concentration in the dilute phase (outside the fibrils) is exponentially low after the fibril formation process, and polymer transport (other than fibril motion) through the solvent phase is prohibited.

\subsection{Solvent release}

Considering the fibril formation process we assumed that fibrils are liquid-like: polymer segments in a fibril are mobile. This means that elongated chains in a fibril should slowly retract with the characteristic time $\tau_{R}^{*}$, the renormalized Rouse time: $\tau_{R}^{*}$ is longer than $\tau_{R}$ since the segmental friction constant $\zeta_{1}^{*}$ in the more dense fibril $\left(\phi \sim \phi_{c}\right)$ is larger than $\zeta_{1}$ in the initial solution with $\phi \sim \phi_{0}$ : $\tau_{R}^{*} \sim\left(\zeta_{1}^{*} / \zeta_{1}\right) \tau_{R}$. Moreover, it is also possible that gliding of elongated chains with respect to each other is arrested in fibrils. Such solidified fibrils are considered in the Discussion (point 6 of section 4). In the current section we continue to consider liquid-like fibrils on time scales $t$ shorter that the fibril degradation time, $t \lesssim \tau_{R}^{*}$

The network of crosslinked fibrils considered in the previous section is swollen by the solvent: the volume fraction of fibrils is small, $\varphi \ll 1$. This is not an equilibrium state as the fibrils attract each other and tend to aggregate further. This process implies that the polymer network must shrink. The system (jet) cannot shrink longitudinally (along the z-axis) due to constraints applied at its ends. Hence, the network must collapse laterally (in the xy plane). This process implies that the pure solvent is released outside the polymer phase, so that the diameter $D_{f}$ of the central polymer fiber decreases gradually. This 'macroscopic' syneresis process is slow (it is considered at the end of this section). An important point is that aggregation of fibrils can be achieved much faster without changing 
the total volume of the polymer fiber: by formation of dense domains of aggregated fibrils (micro-syneresis). As the total volume is conserved, dense domains must alternate with expanded regions (Figure 6). Dense domains necessarily occupy only a small fraction $\sim \varphi \ll 1$ of the volume, yet most of polymer material is concentrated in these regions (polymer bundles extended along $z$-axis with roughly circular cross-section as shown in Figure 6). On the other hand, the dense bundles must be surrounded by the matrix of laterally expanded network of the fibrils. ${ }^{40}$ The stretched matrix therefore dominates by volume. To be more precise, the network deformation is anisotropic also in the xy plane: in a vicinity of a dense domain the network is stretched in the radial direction (away from the bundle), but it is compressed azimuthally.

What is the driving force to network expansion in the matrix? This is actually the energy gained when a network layer adjacent to the bundle/matrix interface collapses onto the bundle. At the mechanical equilibrium this energy gain is balanced by the network elastic energy increment. This balance leads to the following condition: the energy gain as a fibril segment of unit length is sucked into a dense domain,

$$
P \sim T l \phi_{c} / d^{2}
$$

must be comparable with the lateral elastic energy $E_{\boldsymbol{e l}}$ per unit length in the matrix, $E_{e l} \sim F_{t} \theta^{2}$, where $F_{t} \sim n_{f} T / l \sim T /\left(d\left|k_{T}\right|\right)$ is the total longitudinal fibril tension force, $\theta$ is the typical angle between the network strand and the $z$-axis. In the undeformed network $\theta=\theta_{0} \sim \xi / \xi_{z}$, where $\xi$ is its lateral mesh size. For the network stretched laterally (radially) with strain factor $\lambda_{f}$ the angle $\theta$ increases $\theta \simeq \lambda_{f} \theta_{0} \sim \lambda_{f} \xi / \xi_{z}$ (as long as $\theta \ll 1$ ), hence

$$
E_{e l} \sim\left(T l / d^{2}\right) \phi_{0} \lambda_{f}^{2}
$$

Note that network compression in the azimuthal direction does not change much its elastic energy. Thus we get the radial expansion factor and the corresponding angle

$$
\lambda_{f} \sim \sqrt{\phi_{c} / \phi_{0}}, \quad \theta^{2} \sim \phi_{c}\left|k_{T}\right| l / d
$$

Note that for PAN solutions considered in the Appendix $l / d \sim 5$ and $\phi_{c} \sim\left|k_{T}\right| \lesssim 0.1$, so $\theta^{2} \ll 1$.

Based on the geometry depicted in Figure 6, we obtain

$$
D / \Delta \sim \lambda_{f} \sim \sqrt{\phi_{c} / \phi_{0}}, \quad\left(D_{b} / D\right)^{2} \sim \varphi \approx \phi_{0} / \phi_{c}
$$

where $D$ is the distance between the neighboring bundles, $D_{b}$ is their typical thickness, and $\Delta$ is the initial thickness of an undeformed matrix element. Thus $\Delta \sim D_{b} \sim D \sqrt{\varphi}$, and the fraction $f_{p m}$ of polymer material in the matrix is

$$
f_{p m} \sim \Delta / D \sim \sqrt{\varphi} \ll 1
$$

The mean volume fraction of fibrils in the matrix is therefore

$$
\varphi_{p m} \sim \varphi f_{p m} \sim \varphi^{3 / 2}
$$


The bundle size $D_{b}$ increases in time, $D_{b}=D_{b}(t)$. The driving force for this process is the surface energy of dense domains which decreases with $D_{b}$. Note, however, that the total amount of the aggregated polymer is $\propto\left(1-f_{p m}\right)$ and is nearly constant. Thus, the bundle thickening is supposed to occur by Ostwald ripening (Lifshitz-Slezov mechanism ${ }^{38}$ ). In order to obtain the time-dependence of $D_{b}$ it is enough to estimate the time $t$ required to double the domain size, $D_{b} \rightarrow 2 D_{b}$. This can be done by comparing the corresponding energy gain (per unit volume) $\Delta F \sim \gamma_{f} D_{b} / D^{2}$ with the dissipated energy $\mathcal{D} t$, where $\gamma_{f} \sim P / d_{f}$ is the relevant surface tension. The dissipation rate $\mathcal{D}$ is mainly coming from the stretched network strands moving with velocity $v$ (with respect to the solvent) in order to supply additional polymer to the growing dense domains. The current of fibrils (volume of fibrils per unit time) coming to a dense domain is $J \sim v D \varphi_{p m}$. Obviously $J t \sim D_{b}^{2}$ (the increment of domain area is similar to the area itself), so $v \sim D_{b}^{2} /\left(D t \varphi_{p m}\right)$. The total dissipation rate (per unit volume of the system) is $\mathcal{D} \sim \zeta_{\perp} \mathcal{L} v^{2}$, where $\mathcal{L} \sim \varphi_{p m} / d_{f}^{2}$ is the total length of all fibrils in the matrix per unit volume, and $\zeta_{\perp} \sim \zeta_{\|}$. The condition $\Delta F \sim \mathcal{D} t$ leads to the following domain coarsening (micro-syneresis) time:

$$
t=t_{m s} \sim \frac{\eta_{s}}{T k_{H}} \frac{d^{2}}{l d_{f} \phi_{c}} \varphi_{p m}^{-1} D_{b}^{3} \sim \frac{\eta_{s}}{T k_{H}}\left(\frac{d\left|k_{T}\right|}{l \phi_{0}}\right)^{3 / 2} D_{b}^{3}
$$

Therefore, the domain size (bundle thickness) grows as $D_{b} \propto t^{1 / 3}$, i.e. we recover the classical scaling law of domain coarsening ${ }^{38}$. It is remarkable that the microsyneresis process gets faster with increasing the initial polymer concentration $\phi_{0}$, but also as $\left|k_{T}\right|$ decreases, i.e., as $\Theta$-conditions are approached. The above equation can be written as

$$
D_{b} \sim D_{b}^{*}\left(t / \tau_{R}\right)^{1 / 3}, \quad D_{b}^{*} \sim(L / l)^{2 / 3} l^{3 / 2} d^{-1 / 2}\left(\phi_{0} / \phi_{c}\right)^{1 / 2}
$$

Thus, during the Rouse time the bundle diameter increases significantly to $D_{b} \sim$ $D_{b}^{*} \propto N^{2 / 3} \phi_{0}^{1 / 2}$. For $\phi_{0}$ at the dilute/semidilute crossover, $\phi_{0} \propto N^{-1 / 2}$, we find $D_{b}^{*} \propto N^{5 / 12}$, so $D_{b}^{*}$ in this regime is roughly comparable with the coil size $R_{0}$. The typical distance between the neighboring bundles is

$$
D \sim D_{b} / \sqrt{\varphi} \sim(L / l)^{2 / 3} l^{3 / 2} d^{-1 / 2}\left(t / \tau_{R}\right)^{1 / 3}
$$

so at $t \sim \tau_{R}$ the distance between bundles scales as $D \propto N^{2 / 3}$.

Let us now turn to the slow process of solvent release out of the polymer jet (Figure 7). The initial jet diameter is $D_{j}$. Suppose half of the solvent is released. Then the polymer fiber diameter becomes $D_{f}=D_{j} / \sqrt{2}$. The characteristic time of this process, $t_{r}$, is estimated below using the same argument as before (comparing the energy gain with the dissipated energy). As $D_{f}$ decreases, the volume of stretched polymer matrix decreases as well (by a factor of 2) meaning that about half of the matrix polymer can condense into bundles. The corresponding energy gain (per unit volume) is $\Delta E \sim P \mathcal{L}$, where as before $\mathcal{L} \sim \varphi_{p m} / d_{f}^{2}$ is the total length of fibrils in unit volume of the matrix. The dissipation is due friction between the solvent and the matrix: $\mathcal{D} \sim \mathcal{L} \zeta_{\perp} v^{2}$. The typical solvent velocity $v \sim D_{j} / t_{r}$. Hence the dissipated energy $\mathcal{D} t_{r} \sim \mathcal{L} \zeta_{\perp} D_{j}^{2} / t_{r}$. Using the condition $\Delta E \sim \mathcal{D} t_{r}$ we get the solvent release time 


$$
t_{r} \sim \frac{\zeta_{\perp} D_{j}^{2}}{P} \sim \tau_{R}\left(\frac{D_{j} d}{L l}\right)^{2}\left|k_{T}\right|^{-1}
$$

This time is shorter than the Rouse time $\tau_{R}$ if $D_{j} \lesssim L l\left|k_{T}\right|^{0.5} / d$. For PAN of $M_{w}=800 \mathrm{k}$ the latter condition becomes $D_{j} \lesssim 0.01 \mathrm{~mm}$. At $t \gg t_{r}$ the jet appears as a dense fiber with polymer concentration $\approx \phi_{c}$, the diameter $D_{f} \approx D_{j} \sqrt{\phi_{0} / \phi_{c}}$ and an annulus of pure solvent around it with the outer diameter $D_{j}$ (cf. Figure 7).

Note that the length-scale dependencies of the micro-syneresis time $t_{m s}$ and the solvent release time $t_{r}$ are different: $t_{m s} \propto D_{b}^{3}$, while $t_{r} \propto D_{j}^{2}$. Why the scaling laws differ? The reason is that the driving forces for the two phenomena are different: The micro-syneresis is driven by the surface energy which scales as $V / D_{b}$, where $V$ is the total volume. On the other hand, the energy gained by solvent release is the volume energy (scaling as $V$ ) due to increasing fraction of condensed fibrils. Therefore, the second process is faster, the length-scales being equal and large.

\section{Discussion}

1. In this study we considered the phase separation processes generated by strong elongational flows in unentangled polymer solutions $\left(\phi<\phi_{e}\right.$, where $\phi_{e}$ corresponds to the onset of entanglements, cf. the Appendix). The demixing process is driven by the extension-induced attraction between polymer segments ${ }^{21}$ : strong elongational flow (with rate $\dot{\epsilon}$ ) straightens polymer chains due to coil to stretched coil transition ${ }^{31,32}$ which can occur for $\mathrm{Wi}=\dot{\epsilon} \tau_{R}>\mathrm{Wi}_{c} \approx 3.3^{21}$, where $\tau_{R}$ is the chain relaxation time. The chain segments are aligned with orientational order parameter $s \gtrsim 0.7$ upon the transition. This orientation alone results in a reduced excluded-volume repulsion between the segments, so their van-der-Waals attraction can become dominant if the system was originally close to the $\Theta$-conditions (for small $1-\Theta / T$ ). The flow then gives rise to a negative osmotic modulus $\kappa$ (cf. eq. (9)) and hence to the polymer/solvent demixing instability.

Note that $s=R_{z} / L=\langle\cos \theta\rangle$, where $\theta$ is the angle between a polymer segment and the flow direction (cf. Figure 1b), is different from the nematic order parameter: $s$ is induced by the flow rather than by nematic interactions. The nematic transition is not considered here: it is assumed that concentration $\phi \lesssim d / l$ or less.

It may be also useful to comment that the stress-concentration coupling effect (SCC) for the lateral demixing in extensional flows is always weak compared to the orientation-induced thermodynamic (osmotic) driving force for $\mathrm{Wi}>\mathrm{Wi}_{c}$ (i.e., in the regime of stretched chains): To see this, the osmotic pressure $\Pi \simeq T v^{*} c^{2} / 2$ must be compared to the lateral viscous polymer stress $\sigma_{x x} \sim \eta_{x x} \dot{\epsilon}$, where the relevant viscosity $\eta_{x x} \propto R_{x}^{2}$ and $R_{x} \propto 1 / \sqrt{f_{t}}$ is the lateral chain size for $\mathrm{Wi}=\dot{\epsilon} \tau_{R} \gg 1$ ( $f_{t} \propto \dot{\epsilon}$ is the chain tension). Hence, the typical $\sigma_{x x}$ is roughly independent of $\mathrm{Wi}$ for $\mathrm{Wi} \gg 1$, so the lateral SCC driving force is not expected to increase with Wi.

2. The results of this study can be qualitatively applied to several polymer systems like solutions of PAN in DMSO, and PAAA or PEO water solutions. ${ }^{17-20}$ However, in most of the previous experimental studies on extension of very dilute solutions the jet dynamics are strongly affected by the capillary effects related to the Plateau-Rayleigh instability ${ }^{56,57}$ whose interference with possible demixing and 
solvent release processes requires a special consideration. That is why we rather draw attention to semidilute PAN in DMSO solutions ${ }^{17,18}$ where the PlateauRayleigh instability is apparently suppressed. One such system is a PAN solution in $\mathrm{DMSO}^{17}$ with concentration $(\phi \sim 1 \%)$ falling in the semidilute, $\phi>\phi^{*}$, and unentangled, $\phi<\phi_{e}$, regime. Here $\phi^{*}$ is the coil overlap crossover and $\phi_{e}$ is the entanglement onset concentrations discussed in the Appendix together with other relevant properties of PAN solutions.

Generally, the theory is expected to be applicable to high-molecular-weight polymers (with $M \gtrsim 10^{4} \div 10^{6}$ ) in the vicinity of the dilute/semidilute crossover concentration $\phi^{*}$ (in particular, at $\phi \gtrsim \phi^{*}$ ) in the unentangled regime $\left(\phi<\phi_{e}\right)$. The relevant parameters for the reference system considered in the Appendix (solution of PAN in DMSO) are $M \sim 10^{6}$ and $\phi^{*} \sim 0.1 \%$.

3. We distinguished several kinetic stages related to the demixing process: $(0)$ the coil to stretched coil transition; (i) amplification of concentration fluctuations; (ii) polymer fibril and network formation; (iii) microphase separation of the polymer network and eventual solvent release from it.

The theory of spinodal decomposition developed in this paper (stages i-iii) is based on the mean-field approach which is valid in the marginal solvent conditions (where polymer chains are not strongly swollen). Its applicability for a semidilute system, $\phi \sim 1 \%>\phi^{*}$ is justified in the Appendix. It is important that the mean-field theory is also applicable in the dilute regime $\phi<\phi^{*}$ once the chains are stretched. In fact, the Fixman parameter (cf. eq. (A1)) is proportional to $N^{2} / V_{\text {coil }}$, where the coil volume $V_{\text {coil }}$ increases by the factor $\sim L / R_{0}$ on chain stretching. As a result $z_{F}$ strongly decreases for stretched chains becoming independent of $N$ and small: $z_{F} \sim d / l \ll 1$. Hence, the statistics of their lateral fluctuations are nearly Gaussian and the lateral dynamics of stretched chains are essentially Rouse-like. For the same reason the stretched chains can overlap strongly even in the dilute regime $\left(\phi<\phi^{*} \sim v_{1} N / R_{0}^{3}\right)$ provided that $\phi \gg \phi_{\min } \sim \phi^{*} R_{0} / L \sim d^{2} / L l$. For the sake of simplicity we applied a somewhat stronger condition (10), $2 \phi\left|k_{T}\right| L / d \gg 1$, where $k_{T}$ is the effective deviation from the $\Theta$-point. We treat $k_{T}=I(s)-\Theta / T$ as a small parameter assuming that $\left|k_{T}\right| \lesssim 0.1$. This assumption roughly corresponds to the conditions $1-\Theta / T \lesssim 0.1$ (cf. Appendix) and $1-I(s) \lesssim 0.2$ which is true if $s \leq 0.8$. For the PAN solution considered in the Appendix (with $2 L / d \sim N \approx 16000$ ) the condition (10) reduces to $\phi \gg 0.1 \%$.

4. The considered demixing mechanism is hinged on the coil-stretch transition induced by fast enough extension flow. However, the criterion we gave already $\left(\mathrm{Wi}>\mathrm{Wi}_{c}\right.$ ) may seem not to be entirely satisfactory for the following reason: For $\mathrm{Wi}>\mathrm{Wi}_{c}$ the stretched state is indeed both stable and preferred in the quasithermodynamic sense. However, the coil state may be meta-stable (this possibility was emphasized by De Gennes for dilute solutions ${ }^{31,32}$ ). In fact, the coil relaxation time $\tau_{\text {coil }}$ in a dilute solution is the Zimm time, $\tau_{\text {coil }} \sim \tau_{Z} \propto R_{\text {coil }}^{3} \propto N^{3 \nu}$, where the coil size $R_{\text {coil }} \propto N^{\nu}$. In theta- or marginal-solvent conditions $\nu \approx 0.5$ and $\tau_{\text {coil }} \propto N^{1.5}$. On the other hand, the stretched chains are always characterized by the Rouse time $\tau_{R} \propto N^{2}$ (cf. eq. (4)). Hence $\tau_{R} \gg \tau_{\text {coil }}$ for $N \gg 1$, so the condition $\mathrm{Wi}=\dot{\epsilon} \tau_{R}>\mathrm{Wi}_{c}$ may seem to be not sufficient: after a long waiting time the chains would of course overcome the barrier separating the two states (coil and stretched coil), but this would not happen in practice given the necessarily transient nature of any elongational flow: the time a coil spends in such a flow is 
always limited. Hence a stronger condition may be necessary: $\dot{\epsilon} \tau_{\text {coil }} \gtrsim 1$.

Let us apply this condition to the reference system $^{17}$ of PAN in DMSO considered in the Appendix $\left(\phi \sim 1 \%, M_{w} \approx 8.3 \cdot 10^{5}, N=M_{w} / M_{1} \approx 16000\right)$. This solution is actually in semidilute regime since $\phi^{*}(N) \approx 0.13 \%, \phi / \phi^{*}(N) \approx 6.5$, where $\phi^{*}(N)$ is the crossover (coil overlap) concentration for chains with polymerization index $N$ (cf. the Appendix). The polymer coils in this system overlap strongly and are characterized by the Rouse-Zimm time, $\tau_{\text {coil }} \sim \tau_{R Z}$ : Each polymer can be considered as a chain of concentration blobs with $N_{b}$ units per blob (the number of blobs per chain is $n_{b}=N / N_{b}$ ), so that the blobs are at the overlap crossover: $\phi^{*}\left(N_{b}\right)=\phi$. The number $N_{b}=N / n_{b}$ can be obtained using eq. (A6) with $\nu=0.5$ (corresponding to the marginal solvent regime, $\mathrm{cf}$. the Appendix): ${ }^{60}$ $N_{b} \sim N\left(\phi / \phi^{*}\right)^{-2} \sim 400$. The Rouse-Zimm time is then ${ }^{35}$

$$
\tau_{R Z} \approx \frac{\zeta_{b} n_{b} R_{0}^{2}}{3 \pi^{2} T}
$$

where $R_{0} \approx a_{s} \sqrt{N}$ is the coil size, $a_{s}=\sqrt{l_{1} l} \approx 0.8 \mathrm{~nm}$ is statistical segment for PAN $\left(l_{1} \approx 0.25 \mathrm{~nm}\right.$ is contour length per monomer unit, $l \approx 2.5 \mathrm{~nm}$ is Kuhn segment of PAN chain $\left.{ }^{39}\right), \zeta_{b}$ is the blob friction constant calculated with Zimm model ${ }^{35}$ :

$$
\zeta_{b} \approx 5.1 \eta_{s} a_{s} \sqrt{N_{b}}
$$

Thus we get

$$
\tau_{c o i l} \sim \tau_{R Z} \approx 0.17 \frac{\eta_{s}}{T} \frac{N^{2} a_{s}^{2}}{\sqrt{N_{b}}}
$$

and

$$
\tau_{\text {coil }} / \tau_{R} \sim 0.4
$$

where the Rouse time for stretched coil is defined in eq. (4) with hydrodynamic factor $k_{H} \approx 0.5 \ln (1 / \phi) \approx 2.3$. Therefore, $\mathrm{Wi}=\dot{\epsilon} \tau_{R} \sim 4$ corresponds to $\dot{\epsilon} \tau_{\text {coil }} \sim 1.5$. This extension rate is fast enough to initiate stretching of an undeformed coil (i.e., in this case the coil stretching is not hindered by any significant potential barrier). Therefore, the additional condition $\dot{\epsilon} \tau_{c o i l} \gtrsim 1$ is not required for the semidilute system (1\% of PAN in DMSO) considered in ref. 17.

5. It is noteworthy that the predicted critical extension rate $\dot{\epsilon}_{c}=3.3 / \tau_{R}$ (corresponding to $\mathrm{Wi}_{c}=3.3$ ) was derived for semiflexible $(l \gg d)$ persistent-chain polymers. Generally, the numerical prefactor $\left(\mathrm{Wi}_{c}\right)$ depends on the chain flexibility mechanism, but (based on a simple analysis) we expect a deviation to be rather unimportant in practice for most polymers with $l / d \gtrsim 5$. A more important issue was raised in the previous point: the predicted $\dot{\epsilon}_{c}$ corresponds to the onset of the coil-stretch transition in the steady flow conditions. Therefore, in practice the predicted value defines the lower bound for the minimal flow rate $\left(\dot{\epsilon}_{c *}\right)$ required for a fast enough stretching transition. In the semidilute regime $\left(\phi>\phi^{*}\right)$ the difference between $\dot{\epsilon}_{c}$ and $\dot{\epsilon}_{c *}$ is not significant if $\phi / \phi^{*}$ is sufficiently large. In particular, this is true for the system considered in the previous point (the solution of PAN in DMSO, $\left.M \sim 10^{6}, \phi \sim 1 \%\right)$. However, the apparent critical rate $\dot{\epsilon}_{c *}$ is generally a decreasing function of concentration ${ }^{62}$, so $\dot{\epsilon}_{c *}$ can be significantly higher than $\dot{\epsilon}_{c}$ in 
the dilute regime $\left(\phi<\phi^{*}\right)$. Generally, the coil-stretch transition is thus predicted to occur beyond the critical Weissenberg number $\mathrm{Wi}_{c *}=\dot{\epsilon}_{c *} \tau_{R}$,

$$
\mathrm{Wi}_{c \star} \approx \max \left(\mathrm{Wi}_{c}, \frac{\tau_{R}}{\tau_{R Z}}\right)
$$

where $\tau_{R Z}$ is defined in the previous point. Presently we are lacking an experimental verification of the predicted $\mathrm{Wi}_{c}$ since, to our knowledge, all the published data on the critical extension rate related to the coil-stretch transition fall in the dilute solution regime $\left(\phi<\phi^{*}\right)^{62,63}$.

6. After a number of kinetic stages (0)-(ii) involving polymer fibril formation the system transforms into a well-defined network of polymer fibrils (domains of the dense phase). ${ }^{41}$ So far it was assumed that polymer segments are mobile also in the dense phase. It is possible, however, that their long range motions become arrested due to dramatic increase of the segmental friction in the dense environment. This scenario is likely in the case of PAN which gelates at moderate concentrations ${ }^{43,44}$. In fact, an extrapolation of the experimental data on PAN gelation in $\mathrm{DMSO}^{43,44}$ leads to bulk gelation at $\phi \sim 5 \%$ at room $T$. The polymer concentration in the dense phase is $\phi_{c} \simeq(T / \Theta)\left|k_{T}\right| \simeq\left|k_{T}\right| \sim 0.1$, so its gelation is expected. In this case the mature fibrils and their bundles, once formed, preserve their structure: the strongly stretched polymer chains inside these filaments do not relax and remain aligned forever, so the condition $t \lesssim \tau_{R}^{*}$ applied in the previous section becomes irrelevant. It is then expected that the fibrils are solidlike, hence, their rigidity segment increases: $l_{f} \sim \ln _{f}^{2}$ (cp. eq. (28)). Note that the number of polymer chains per fibril is expected to be moderately large: for PAN in DMSO solutions with $\left|k_{T}\right| \sim 0.1$ eq. (18) gives: $n_{f} \sim \frac{3}{\left|k_{T}\right|} \sim 30$.

Otherwise not much is changed in the fibrillar network dynamics: the main energy dissipation process is related to viscous friction between the solvent and the network strands which remain nearly straight both with liquidlike and solidlike fibrils. Thus, all the results obtained in the previous section are applicable in both cases. The only change may concern the fibril diameter and density: if the equilibrium concentration $\phi_{c}$ is not accessible for kinetic reasons, it must be replaced by the gelation concentration $\phi_{g}$. In this case $\left(\phi_{g}<\phi_{c}\right)$ the fibril diameter is larger: $d_{f} \sim \xi \sqrt{\phi_{0} / \phi_{g}}$ (cp. eq. (27)). It is noteworthy that $\phi_{g}$ in the confined fibril geometry may be different from the bulk gelation concentration.

7. In the case of fibril gelation the fibril network structure remains intact even after the solvent release from the polymer phase. No matter how long the solvent release process is, it should result in formation of a strong crosslinked and solidlike fiber. Its permanent network/microdomain structure necessarily implies that the fiber density is significantly inhomogeneous in its cross-section. This prediction is in agreement with experimental data on strong light-scattering of stretched PAN/DMSO polymer jets ${ }^{18}$. Moreover, formation of such solidlike and unstretchable fiber (say, in the middle of the jet) may lead to faster elongation of the neighboring jet parts, hence a fast propagation of phase separation along the jet is expected. This conclusion is also in agreement with experimental data ${ }^{18}$. In the case of systems with solidified fibrils one is tempted to expect that the solvent release process can be accelerated by applying a strong enough elongational stress $\sigma_{z z}$, if the applied mechanical tension per fibril network strand exceeds its elastic tension $F_{t}$ (cf. eq. (26)): $\sigma_{z z} \gtrsim F_{t} / \xi^{2} \cdot{ }^{45}$ 
8. The early stage of extension-induced spinodal decomposition was studied in section 3.1. In order to simplify the model we assumed an instantaneous jet elongation by a large deformation factor increasing the longitudinal chain size from $R_{\text {coil }} \sim R_{0} \propto N^{0.5}$ to $R_{z} \approx s L \propto N$. A more realistic continuous elongation with constant extension rate $\dot{\epsilon}$ is considered below. The reduced extension rate $U=2 \dot{\epsilon} \tau_{R} \equiv 2 \mathrm{Wi}$ must be fast enough: $U>U_{c}=2 \mathrm{Wi}{ }_{c}$. In addition, the osmotic modulus $\kappa$ in the stationary regime with $s=s(U)$ must be negative. As $\kappa \propto I(s)-\Theta / T$, the latter condition boils down to $I(s(U))<\Theta / T$, where the functions $s(U)$ and $I(s)$ are defined in eq. (5) and eq. (7), respectively. Hence, we arrive at the condition $U>U^{*}$, where $U^{*}$ is defined by $I\left(s\left(U^{*}\right)\right)=\Theta / T$. The chain stretching rate $s^{-1} \mathrm{~d} s / \mathrm{d} t$ comes as a result of extension with rate $U$ and retraction with rate $U(s)$, where $U(s)$ is the inverse function of $s(U)$ :

$$
s^{-1} \mathrm{~d} s / \mathrm{d} t=(U-U(s)) /\left(2 \tau_{R}\right)
$$

The growth of concentration fluctuations starts in the transient regime as soon as $s$ exceeds $s^{*} \equiv s\left(U^{*}\right)$ corresponding to $\kappa=0$. For small $s-s^{*}$ eq. (33) yields $s-s^{*} \simeq B t$, where $B=\frac{s^{*}\left(U-U^{*}\right)}{2 \tau_{R}}$ and $t$ is the time passed since the critical stretching degree $s=s^{*}$ was attained. The kinetic equation (16) then modifies as

$$
\begin{gathered}
\frac{\mathrm{d} \ln c_{q}}{\mathrm{~d} t} \simeq \Gamma(q, t) \\
\Gamma(q, t) \simeq \frac{T}{\zeta_{1}} q^{2}\left(2 \phi_{0} \frac{l_{1}}{d} \beta t-\frac{q^{2} a^{2}}{2}-\frac{q_{z}^{2} s^{2} l_{1}^{2}}{2 q^{2} a^{2}}\right)
\end{gathered}
$$

where $\beta=\left(\frac{\mathrm{d} I(s)}{\mathrm{d} s}\right)_{s^{*}} B=s^{*}\left(\frac{\mathrm{d} I(s)}{\mathrm{d} s}\right)_{s^{*}}\left(\dot{\epsilon}-\dot{\epsilon}^{*}\right)$ and $\dot{\epsilon}^{*}=U^{*} /\left(2 \tau_{R}\right)$. The corresponding characteristic growth time $\tilde{t}_{l}$, and lateral $(\tilde{\xi})$ and longitudinal $\left(\tilde{\xi}_{z}\right)$ length-scales are

$$
\begin{gathered}
\tilde{t}_{l} \sim t_{l 0}\left(\beta t_{l 0}\right)^{-2 / 3}, \quad t_{l 0} \sim \tau_{R} /\left(\phi_{0} L / d\right)^{2} \\
\widetilde{\xi} \sim\left(\frac{l d}{\phi_{0}}\right)^{1 / 2}\left(\beta t_{l 0}\right)^{-1 / 6}, \quad \tilde{\xi}_{z} \sim \frac{d}{\phi_{0}}\left(\beta t_{l 0}\right)^{-1 / 3}
\end{gathered}
$$

Thus, the growth time is proportional to $\left(\dot{\epsilon}-\dot{\epsilon}^{*}\right)^{-2 / 3}$, the lateral length $\tilde{\xi} \propto$ $\left(\dot{\epsilon}-\dot{\epsilon}^{*}\right)^{-1 / 6}$, and the longitudinal length $\tilde{\xi}_{z} \propto\left(\dot{\epsilon}-\dot{\epsilon}^{*}\right)^{-1 / 3}$, so all the time/length scales decrease with the extension rate $\dot{\epsilon}-\dot{\epsilon}^{*}$. Eqs. (35), (36) are valid for $\beta \tilde{t}_{l} \ll k^{*}$, where $k^{*}=\left(\frac{\mathrm{d} I(s)}{\mathrm{d} s}\right)_{s^{*}}\left(1-s^{*}\right)$. The latter condition can be represented as

$$
\mathrm{Wi}=\dot{\epsilon} \tau_{R} \ll\left(\phi_{0} L / d\right)^{2} k^{* 3}
$$

The above condition allows for high Weissenberg numbers since $\left(\phi_{0} L / d\right)^{2} \gg 1$. The condition (37) also ensures that the growth time $\tilde{t}_{l}$ is much shorter than the extension time $1 / \dot{\epsilon}$. Therefore, the advection effects (that have been neglected in eq. (34)) are indeed small. At high extension rates, $\mathrm{Wi} \sim\left(\phi_{0} L / d\right)^{2} k^{* 3}$, the results, eqs. (35), (36), smoothly cross over to the predictions $\left(t_{l}, \xi, \xi_{z}\right)$ derived in section 3.1 for instantaneous extension (with $s \sim\left(1+s^{*}\right) / 2$ ). 
9. The spinodal decomposition considered in the previous point proceeds in parallel with chain stretching. Moreover, the characteristic concentration wave growth time $\tilde{t}_{l}$ is shorter than the stretching time $1 / \dot{\epsilon}$ in the regime considered above. In this case the chains have enough time to relax on the relevant lengthscales (lateral, $\xi$, and longitudinal, $\xi_{z}$ ) before the growth process sets in. This feature justifies the validity of the quasi-thermodynamic approach (taken to analyze the growth process) involving the free energy $\mathcal{F}$ considered as a functional of the concentration field $c(\underline{r})$ (cf. eq. (14)).

However, in the main part, section 3.1, we considered the extremely opposite case of instantaneous stretching. The growth process takes place after the stretching, so the lateral relaxation of chains has to proceed during the growth process. Yet, the quasi-thermodynamic approach is still valid (with logarithmic accuracy) also in this case since the relevant chain relaxation time (the lateral spreading time for a chain fragment of length $\xi_{z}$ ) is $\sim 1 / \Gamma^{*}$ while the growth time is longer, $t_{l} \sim\left(0.5 \ln n_{f}\right) / \Gamma^{*}$ (cf. eq. (19)). Note also that in section 3.1 the thermal noise was accounted for in the initial conditions, but neglected in the dynamical equation (11). This approximation leads to a similar logarithmically small error (for the growth time).

10. The theory is quantitatively applicable to semiflexible polymers with Kuhn segment $l$ significantly longer than the polymer chain thickness $d$. This condition is not restrictive, it is valid for most polymers. For example $l / d \sim 5$ for PAN in DMSO (cf. the Appendix), which is the polymer solution considered as a reference system in our study. On the other hand, the purely nematic orientational effects are negligible at low concentrations $(\phi \lesssim d / l$ or less) considered here. The latter condition is not important as it is always valid in the unentangled regime $\phi<\phi_{e}$ for the relevant polymers studied experimentally (with not too large ratio $l / d)$. In principle, for $\phi \gtrsim d / l$ the nematic interactions would increase the segment orientation degree and therefore would further strengthen the phase separation effects.

11. Our last point concerns the jet extension experiments reported in ref. 17 (solution of PAN in DMSO, $\phi \sim 1 \%, N \approx 16000$ ): Right before the jet was elongated enough to observe the demixing effects $\left(t<t^{*}\right)$, its diameter was $D_{j} \approx 0.13 \mathrm{~mm}$ and length $L_{j} \approx 75 \mathrm{~mm}$. The time of Plateau-Rayleigh capillary instability ${ }^{56,57}$ for the jet is $\tau_{v i s} \approx 3 \eta D_{j} / \gamma$, where $\eta$ is the solution viscosity, $\gamma \approx 43.5 \mathrm{dyn} / \mathrm{cm}$ is surface tension of DMSO (inertial effects are negligible in this case). The viscosity of this solution (with $\vartheta \equiv c[\eta] \approx 4.65$, cf. Supplemental to ref. 17) was estimated using Martin equation ${ }^{55}$ (with numerical prefactor 0.23 in the exponent $\left.{ }^{46}\right): \quad \eta \approx 15 \eta_{s} \approx 0.3 \mathrm{P}$. The predicted jet life-time $\tau_{v i s} \approx 0.3 \mathrm{~ms}$ appears to be much shorter than the experimental elongation time $t^{*} \sim 60 \mathrm{~ms}$. The observed jet stability for $t<t^{*}$ can be explained only by a significant increase of its effective (longitudinal) viscosity by a factor $\sim 100$. In our view, such a viscosity increase during jet extension is indicative of significant coil stretching which is known to be the basic mechanism for extension thickening in unentangled polymer solutions ${ }^{64,31,47-49}$. We therefore conclude that the coil to stretched coil transition have been indeed generated in the jet extension experiments of ref. 17. It appears that what they observe after about $60 \mathrm{~ms}$ of extension for the $1 \%$ solution (oriented fiber formation with solvent separated on its surface $)^{17}$ corresponds the late stage of solvent release. 
The predicted scenario of extension-induced phase separation qualitatively agrees also with the sequence of events observed in ref. 18 for a semidilute PAN/DMSO solution (strong extension $\rightarrow$ jet thinning $\rightarrow$ solvent release $\rightarrow$ highly oriented solidified fiber). Note however that the experimental system studied in ref. 18 was more concentrated (entangled). More efforts on both theoretical and experimental sides would be required to elucidate the demixing effects in such systems.

\section{Conclusions}

1. In this paper we present a theoretical analysis of demixing phenomena generated by uniaxial elongational flows in unentangled high-molecular-weight polymer solutions. The theory is based on the approach proposed in ref. 21. In short, the flow-induced turbidity effects result from (a) polymer coil conformational transition to a strongly stretched state, (b) significant alignment of chain segments along the extension axis, (c) the associated decrease of the excluded-volume steric repulsion of segments leading to their net attraction due to van-der-Waals interactions.

2. The coil to stretched coil transition is predicted if the extension rate $\dot{\epsilon}$ exceeds some critical value: $\mathrm{Wi}>\mathrm{Wi}_{c} \approx 3.3$, where $\mathrm{Wi}=\dot{\epsilon} \tau_{R}$ is the relevant Weissenberg number. The stretched coil is characterized by the essentially Rouse relaxation time $\tau_{R} \propto L^{2}$, where $L$ is the chain contour length. The subsequent polymer/solvent demixing process is driven by the negative polymer osmotic pressure resulting from chain stretching. The osmotic pressure is nearly proportional to the factor $k_{T}=I(s)-\Theta / T$, where $\Theta$ is the theta-temperature, and $I(s)$ is a decreasing function of the orientational order parameter $s=\langle\cos \theta\rangle$ (cf. Figure 1b). Therefore, $k_{T}$ becomes negative as $s$ is increased. Then, the eventual value of $\left|k_{T}\right|$ defines the time and length scales of the demixing structure (cf. eqs. (20), (17), (21)).

3. The following main kinetic stages of the extension-induced spinodal decomposition process are predicted in this study for $\left|k_{T}\right| \phi_{0} L / d \gg 1$, where $\phi_{0}$ is the mean polymer volume fraction in the initial solution:

(i) The early stage: amplification of concentration waves by anisotropic spinodal decomposition (formation of oriented protofibrils).

(ii) The intermediate stage: fibril and network formation. Lateral collapse of fibrils and their densification occur during this stage. A network (cross-linked fiber) of well-defined highly-oriented and stiff fibrils is developed as a result. The predicted number of chains per fibril (network strand) is large: $n_{f} \gtrsim(l / d) /\left|k_{T}\right|$.

(iii) The late stage involves two processes: formation of bundles of fibrils in the crosslinked polymer fiber (its transient microphase separation) and solvent release from the fiber. The polymer phase collapses laterally as a result yielding strong dense and highly oriented fiber surrounded by an annulus of pure solvent. Gelation effects may render the fiber solidlike with indefinite lifetime in agreement with experimental findings ${ }^{17,18}$.

4. In the case of an instant extension the characteristic time of the early stage $t_{l} \sim \tau_{R} /\left(\left|k_{T}\right| \phi_{0} L / d\right)^{2} \sim \tau_{m} /\left(\left|k_{T}\right| \phi_{0}\right)^{2}$ is much shorter than the Rouse time $\tau_{R}\left(\tau_{m}\right.$ is a monomer time). The concentration pattern of the early stage is characterized by two length-scales: the lateral length $\xi \sim(l d)^{0.5} /\left(\left|k_{T}\right| \phi_{0}\right)^{\mathbf{0 . 5}}$ (distance between the protofibrils) and the longitudinal correlation length $\xi_{z} \sim d /\left|k_{T}\right| \phi_{0}$. Both lengths are much shorter than the coil size $R_{\text {coil }}$. In the case of continuous extension the structural parameters depend on the extension rate. The following scaling laws 
are predicted for high Weissenberg numbers, Wi $\gg 1$, in this case: $t_{l} \propto \mathrm{Wi}^{-2 / 3}$, $\xi \propto \mathrm{Wi}^{-1 / 6}, \xi_{z} \propto \mathrm{Wi}^{-1 / 3}$ (cf. point 8 of section 4 for more details). The late-stage time of the solvent release process, $t_{r} \sim \tau_{R}\left(\frac{D_{j}}{L}\right)^{2}$, depends on the jet diameter $D_{j}$. The time $t_{r}$ is longer than the Rouse time if $D_{j}$ exceeds the polymer contour length. The bundle thickness grows as $D_{b} \propto t^{1 / 3}$ reaching $D_{b} \propto \phi_{0}^{1 / 2} D_{j}^{2 / 3}$ at the end of the solvent release stage. Therefore, the bundles are always thinner than the whole fiber, $D_{b} \ll D_{j}$. The predicted bundle structure is in accord with the experimental findings pointing to a strongly inhomogeneous cross-section of the polymer fiber emerging during the solvent release process ${ }^{18}$.

5. The mean-field theoretical approach developed in this paper is valid in a rather wide concentration range: $\phi_{\min } \ll \phi<\phi_{e}$, where $\phi_{e}$ is the entanglement onset concentration (cf. the Appendix), and $\phi_{\min } \sim d^{2} / L l$. Therefore $\phi_{\min } \propto 1 / N$ can be much lower than the coil overlap concentration $\phi^{*} \propto N^{1-3 \nu}$ for large polymerization index $N$, i.e., the theory can be applicable also to dilute solutions. This feature is related to the fact that the degree of coil overlap strongly increases on chain stretching (cf. section 2).

\section{Acknowledgements}

We acknowledge a partial support from the International Research Training Group (IRTG) "Soft Matter Science: Concepts for the Design of Functional Materials". A.V.Subbotin acknowledges financial support from the Russian Science Foundation, Grant \# 14-23-00003.

\section{APPENDIX A: Some definitions and physical properties of PAN solutions}

\section{Marginal solvent regime.}

The size (mean-square end-to-end distance) of an ideal chain is $R_{0}=a_{s} N^{1 / 2}$, where $N$ is polymerization index and $a_{s}$ is the statistical segment length. The chain size $R_{\text {coil }}$ in good solvent is larger, $\alpha=R_{\text {coil }} / R_{0}>1 ; R_{\text {coil }} \propto N^{\nu}$ for long chains with $\nu>0.5$, so the expansion factor $\alpha$ can become however large. The opposite case when $\alpha$ is close to 1 , say $|\alpha-1| \lesssim 0.2$, is referred to as marginal(or theta-) solvent regime ${ }^{30}$. The self-consistent mean-field theoretical approaches are valid in these latter regimes. The swelling factor $\alpha$ is defined by the Fixman parameter ${ }^{5-52}$

$$
z_{F}=\left(\frac{3}{2 \pi}\right)^{3 / 2} \frac{v N^{1 / 2}}{a_{s}^{3}}
$$

where $v$ is the excluded volume per monomer residue, and $\alpha \lesssim 1.2$ roughly corresponds to $z_{F} \lesssim 0.5$. Using this criterion for PAN in DMSO we get

$$
N \lesssim \widetilde{N} \sim 700
$$

The following parameters of PAN are used here: $a_{s}=\sqrt{l_{1} l} \approx 8 \AA \quad\left(l_{1}=L / N \approx 2.5 \AA\right.$ is the contour length per monomer, $l \approx 25 \AA$ is Kuhn segment for $\left.\mathrm{PAN}^{39}\right), v \approx 30 \AA^{3}$ 
(based on the experimental data of ref. 39 for the second virial coefficient $A_{2}$; note that $A_{2} \simeq 0.5 v N_{A} / M_{1}^{2}$ in the short-chain limit, where $M_{1} \approx 53 \mathrm{~g} / \mathrm{mol}$ is molecular weight per unit). Note that the effective thickness of PAN chain is $d \approx 5 \AA$ based on van-der-Waals volume per monomer $\approx 55 \AA^{353}$.

\section{Crossover concentration.}

The crossover (coil overlap) concentration of a polymer solution can be defined $\operatorname{as}^{54}$

$$
c^{*}=0.77 /[\eta]
$$

where $[\eta]$ is the intrinsic viscosity of the solution. In the marginal solvent regime $[\eta]$ is predicted by the Zimm theory ${ }^{\mathbf{3 5}}$ :

$$
[\eta] \approx 0.425 R_{\text {coil }}^{3} / N
$$

with $R_{\text {coil }} \simeq R_{0}$ for $N \gg 1$, hence

$$
\phi^{*} \equiv v_{1} c^{*} \approx 1.8 \frac{v_{1}}{a_{s}^{3}} N^{-1 / 2}
$$

where $v_{1}=M_{1} /\left(N_{A} \rho_{u}\right)$ and $\rho_{u}$ is the density of undiluted polymer.

Entanglement onset concentration.

Definition of concentration corresponding to the onset of entanglements in a semidilute polymer solution is rather an issue ${ }^{55}$. Here we use a simple recipe to estimate it: Following De Gennes ${ }^{32}$ consider the solution as a melt of concentration blobs. For $\phi>\phi^{*}$ the number of blobs per chain, $n_{b}$ scales as $n_{b} \propto \phi^{1 /(3 \nu-1)}$; $n_{b} \sim 1$ for $\phi=\phi^{*} \propto N^{1-3 \nu}$, hence

$$
n_{b} \sim\left(\phi / \phi^{*}\right)^{1 /(3 \nu-1)}
$$

It is reasonable to assume that the polymer is entangled if $n_{b}>N_{e}$, where $N_{e}$ is number of units per entanglement in the polymer melt. This criterion leads to $\phi_{e} \sim \phi^{*} N_{e}^{3 \nu-1}$, which for $\nu \approx 0.5$ (nearly $\Theta$-conditions) yields

$$
\phi_{e} \sim \phi^{*} N_{e}^{0.5}
$$

\section{Some properties of $1 \%$ semidilute PAN/DMSO solution.}

The following system was studied rheologically in ref. 17: solution of PAN with molecular weight $M_{w} \approx 8.3 \cdot 10^{5}$, polymerization index $N=M_{w} / M_{1} \approx 16000$ and polymer concentration $\rho \approx 0.01 \mathrm{~g} / \mathrm{cm}^{3}$ corresponding to $\phi \approx \rho / \rho_{u} \approx 0.85 \%$ $\left(\rho_{u} \approx 1.18 \mathrm{~g} / \mathrm{cm}^{3}\right.$ is density of undiluted PAN). Its intrinsic viscosity ${ }^{17}$

$$
[\eta]_{\rho} \equiv \frac{N_{A}}{M_{1}}[\eta] \approx 465 \mathrm{~cm}^{3} / \mathrm{g}
$$

corresponds to the coil overlap concentration $\rho^{*} \approx \frac{0.77}{[\eta]_{\rho}} \approx 0.0016 \mathrm{~g} / \mathrm{cm}^{354}$, so $\phi^{*} \approx$ $0.13 \%$. Thus, $\phi / \phi^{*} \approx 6.5$, and the solution falls in the semidilute regime. On the other hand, the entanglement onset is $\phi_{e} \sim \sqrt{N_{e}} \phi^{*} \approx 8 \phi^{*}$ with $N_{e}=M_{e} / M_{1} \approx 60$ for $\mathrm{PAN}^{53}$. Hence $\phi<\phi_{e}$ : the polymer solution is expected to be unentangled.

Using experimental intrinsic viscosity, eq. (A8), and eq. (A4) it is possible to independently find the coil size $R_{\text {coil }}$. Comparing it with $R_{0}=a_{s} N^{0.5}, a_{s} \approx 8 \AA$ we get the expansion coil expansion factor $\alpha=R_{\text {coil }} / R_{0} \approx 1.14$. This estimate indicates 
that the chains are swollen just slightly, i.e., they are in the marginal solvent regime.

Another way to get the same conclusion is to apply the criterion (A2), taking into account that the relevant chain fragment corresponds to the concentration blob of $N_{b}=N / n_{b}$ units. With $n_{b} \sim\left(\phi / \phi^{*}\right)^{2}$ (cf. eq. (A6) with $\nu=0.5$ ) we get $N_{b} \sim 400<\widetilde{N} \sim 700$, hence the coil expansion is weaker than $\sim 20 \% .{ }^{61}$

The weak coil expansion is in harmony with the fact that the experimental room temperature $T$ is close to the $\Theta$-temperature for PAN solutions. In fact, $\Theta \approx 11^{\circ} C$ was reported for PAN solution of $c=7.5 \%{ }^{18}$. Hence $1-\Theta / T \lesssim 0.1$ at room $T$. 


\section{REFERENCES AND NOTES}

${ }^{1}$ R.G. Larson, Rheol. Acta 1992, 31, 497-520.

2 J.W. van Egmond, G. G. Fuller, Macromolecules 1993, 26, 7182-7188.

${ }^{3}$ E. Helfand, G.H. Fredrickson, Phys. Rev. Lett. 1989, 62, 2468-2471.

${ }^{4}$ M. Doi, A. Onuki, J. Phys. II France 1992, 2, 1631-1656.

${ }^{5}$ S.T. Milner, Phys. Rev. E 1993, 48, 3674-3691.

${ }^{6}$ M. Cromer, M.C. Villet, G.H. Fredrickson, L.G. Leal, R. Stepanyan, M.J.H. Bulters, J. Rheol. 2013, 57, 1211-1235.

${ }^{7}$ A. Silberberg, W. Kuhn, Nature 1952, $170,450$.

${ }^{8}$ J. Elliasaf, A. Silberberg, A. Katchalsky, Nature 1955, 176, 1119.

${ }^{9}$ M. Tirrell, Fluid Phase Equilib. 1986, 30, 367.

${ }^{10}$ F.C. Frank, A. Keller, M.R. Mackley, Polymer 1971, 12, 467-473.

${ }^{11}$ C. Rangel-Nafaile, A. B. Metzner, K. F. Wissbrun, Macromolecules 1984, 17, 1187-1195.

${ }^{12}$ P. J. Barham, A. Keller, Macromolecules 1990, 23, 303-309.

${ }^{13}$ J. Yerushalmi, S. Katz, R. Shinnar, Chem. Eng. Sci. 1970, 25, 1891.

${ }^{14}$ M. E. Helgeson, L. Porcar, C. Lopez-Barron, N. J. Wagner, Phys. Rev. Lett. 2010, 105, 084501.

${ }^{15}$ M. Cromer, M. C. Villet, G. H. Fredrickson, and L. G. Leal, Phys. Fluids 2013, 25, 051703.

${ }^{16}$ A.Onuki, Phys.Rev.Lett. 1989, 62, 2472-2475.

${ }^{17}$ A.V. Semakov, I.Yu. Skvortsov, V.G. Kulichikhin, and A.Ya. Malkin, JETP Lett. 2015, 101, 690-692.

${ }^{18}$ A.V. Semakov, V.G. Kulichikhin, A.K. Tereshin, S.V. Antonov, A.Ya. Malkin, J. Polym. Sci. B: Phys Ed. 2015, 53, 559-565.

${ }^{19}$ R. Sattler, C. Wagner, J. Eggers, Phys. Rev. Lett. 2008, 100, 164502.

${ }^{20}$ R. Sattler, S. Gier, J. Eggers, C. Wagner, Phys. Fluids 2012, 24, 023101.

${ }^{21}$ A.V.Subbotin, A.N.Semenov, J. Polym. Sci. B: Phys. Ed. 2016, 54, 1066.

${ }^{22}$ A. V. Bazilevskii, S.I. Voronkov, V.M.Entov, A.N. Rozhkov, Sov. Phys. Dokl. 1981, 26, 333-336.

${ }^{23}$ M. S. N. Oliveira, G. H. McKinley, Phys. Fluids 2005, 17, 071704.

${ }^{24}$ A brief preliminary discussion of these problems can be found in ref. ${ }^{25}$.

25 A.N. Semenov, A.V. Subbotin, AIP Conf. Proc. 2016, 1736, 020086.

${ }^{26}$ K. de Moel, E. Flikkema, I. Szleifer, G. ten Brinke, Europhys. Lett. 1998, 42, 407-412.

${ }^{27}$ This approximation is discussed and justified in the Appendix; cf. ref. 30 for its more general discussion. It is noteworthy that this approximation is not important for most of the results obtained in this study, cf. points 3 and 4 of section 4.

${ }^{28}$ A.R. Khokhlov, Polymer 1978, 19, 1387.

${ }^{29}$ D. Schaefer, J.-F. Joanny, P. Pincus, Macromolecules 1980, 13, 1280.

${ }^{30}$ A. N. Semenov, A. A. Shvets, Soft Matter 2015, 11, 8863 - 8878.

${ }^{31}$ P. G. De Gennes, J. Chem. Phys. 1974, 60, 5030-5042.

${ }^{32}$ P.-G. de Gennes, Scaling Concepts in Polymer Physics; Cornell Univ. Press: Ithaca, 1979.

${ }^{33}$ A.N. Semenov, A.R. Khokhlov, Sov. Phys. Usp. 1988, 156, 988-1014.

${ }^{34}$ The function $I(s)$ is parametrically defined (with parameter $A$ ) by: $s=\operatorname{coth}(A)-$ $1 / A, I=2 I_{2}(2 A) / \sinh ^{2}(A)$, where $I_{2}$ is an Infeld function. 
${ }^{35}$ M. Doi, S. F. Edwards, The Theory of Polymer Dynamics; Oxford Univ. Press: New York, 1986.

${ }^{36}$ Note that coupling between $\delta c$ and the fluctuations $\delta s$ of the orientational order parameter $s$ can be neglected as long as tension fluctuations $\delta f$ are weak compared with the mean chain tension corresponding to $s$. This is true if $\phi_{0} l / d \ll 1$. The latter condition is assumed.

${ }^{37}$ The random thermal force is neglected in this equation: The effect of this force is equivalent to an unimportant enhancement (by a numerical factor $\sim 2$ ) of initial amplitudes of the relevant thermal fluctuation modes.

${ }^{38}$ I.M. Lifshitz, V.V. Slyozov, J. Phys. Chem. Solids 1961, 19, 35.

${ }^{39}$ K.Kamide, H.Yamazaki, Y.Miyazaki, Polym. J. 1986, $18,819$.

40 The (rather local) process of bundle nucleation is not considered here.

${ }^{41}$ Such structure formation had been anticipated already in an early study ${ }^{42}$.

${ }^{42}$ S.Ya. Frenkel, Ukr. Fiz. Zh. 1967, 12, 282-289.

${ }^{43}$ Lianjiang Tan, Shuiping Liu, Ding Pan J. Phys. Chem. B 2009, 113, 603-609.

${ }^{44}$ Lianjiang Tan, Ding Pan, Ning Pan, Polymer 2008, 49, 5676-5682.

${ }^{45}$ A more detailed analysis (akin to that given in section 3.3) shows however that the effect of $\sigma_{z z}$ is weak unless $\sigma_{z z} \gtrsim\left(F_{t} / \xi^{2}\right)\left(\phi_{c} / \phi_{0}\right)$, i.e., a much higher stress is required for the acceleration.

${ }^{46}$ S.O. Ilyin, E.V. Chernikova, Yu.V. Kostina, V.G. Kulichikhin, A.Ya. Malkin, Polymer Science, Ser. A 2015, 57, 494-500.

${ }^{47}$ F. Durst, R. Haas, B.U. Kaczmar, J. Appl. Polym. Sci. 1981, 26, 3125.

${ }^{48}$ G. Chauveteau, M. Moan, A. Magueru, J. Non-Newtonian Fluid Mech. 1984, 16, 315.

${ }^{49}$ A.J. Mueller, J.A. Odell, A. Keller, J. Non-Newt. Fluid Mech. 1988, 30, 99-118.

${ }^{50}$ I.M. Lifshitz, A.Yu. Grosberg, A.R. Khokhlov, Rev. Mod. Phys. 1978, 50, 683.

${ }^{51}$ A. Grosberg, A. Khokhlov, Statistical Physics of Macromolecules; American Institute of Physics: New York, 1994.

${ }^{52}$ A.N. Semenov, I.A. Nyrkova, Statistical Description of Chain Molecules, in Polymer Science: A Comprehensive Reference, ed. K. Matyjaszewski and M. Moeller, Elsevier BV, Amsterdam 2012, 1, 3-29.

53 http://polymerdatabase.com/polymers/polyacrylonitrile.html

${ }^{54}$ W.W. Graessley, Polymer 1980, 21, 258.

${ }^{55}$ W.W. Graessley, The entanglement concept in polymer rheology; Adv. Polym. Sci. 1974, 16, 1.

${ }^{56}$ L. Rayleigh, Proc. Lond. Math. Soc. 1878, 1, 4.

${ }^{57} \mathrm{C}$. Weber, $Z A M M$ 1931, 11, 136.

${ }^{58}$ J.W. Cahn, J.E. Hilliard, J. Chem. Phys. 1958, 28, 258-267.

59 J.W. Cahn, J. Chem. Phys. 1959, 30, 1121; J. Chem. Phys. 1965, 42, 93.

${ }^{60}$ Actually the PAN chains may be slightly swollen in DMSO at room $T$ (cf. the end of Appendix). For the given $\phi$ and $\phi^{*}$ the swelling leads to a somewhat lower $n_{b}$ (cf. eq. (A6)), but it also makes the ratio of the genuine Rouse time to Zimm time lower for a given $N_{b}=N / n_{b}$. The two effects nearly compensate each other, so the final result for $\tau_{R Z}$ stays the same even with some PAN chain swelling.

${ }^{61}$ We also would like to state that this conclusion is in agreement with experimental data for the Mark-Kuhn-Houwink exponent $3 \nu-1 \approx 0.6^{39}, 3 \nu-1 \approx 0.62$ (based on supplemental data ${ }^{17}$ ), while the data of ref. 46 point to a somewhat stronger 
swelling, $3 \nu-1 \approx 0.68$.

${ }^{62}$ A. Keller, J.A. Odell, Colloid Polym. Sci. 1985, 263, 181-201.

${ }^{63}$ C.A. Farrell, A. Keller, M.J. Miles, D.P.Pope, Polymer 1980, 21, 1292-1294.

${ }^{64}$ R.B. Bird, R.C. Armstrong, O.Hassager, Dynamics of Polymeric Fluids; Wiley: NY, 1987. 


\section{FIGURE CAPTIONS}

FIG. 1. (a) Equilibrium coil of size $R_{\text {coil }} \propto N^{0.5}$ and a stretched chain of size $R_{z} \propto N$. (b) The chain is extended by the pulling friction force $\Delta H, R_{z}=L s$, $L=N l_{1}, s=\langle\cos \theta\rangle$ is the orientational order parameter.

FIG. 2. (a) The typical transient partially demixed structure resulting from the growing concentration waves. An xy cross-section (perpendicular to the extension axis) is shown. Note an overall symmetry between black $(\delta c>0)$ and white $(\delta c<0)$ regions. (b) The more dense domains are strongly anisotropic in the $\mathrm{xz}$ plane; $\xi$ is their lateral spacing, $\xi_{z}$ is the longitudinal correlation length.

FIG. 3. (a) An xy cross-section of the domain structure during the intermediate stage of fibril formation. (b) Network of stiff oriented polymer fibrils of thickness $d_{f} \ll \xi$.

FIG. 4. Two chains neighboring inside a fibril at $z=z_{1}$ become separated at $z=z_{2}$.

FIG. 5. A part of a fibril of diameter $d_{f}=d_{f}(t)$.

FIG. 6. (a) On the right: Inhomogeneous network of fibrils with microdomain pattern including fibril bundles (of diameter $D_{b}$ ) surrounded by the laterally expanded matrix of crosslinked fibrils. The structure in the xy plane is shown. The distance between the neighboring bundles is $D$. On the left: The initial homogeneous undeformed network of fibrils (before the bundle formation): the grey domains incorporate fibrils that would form bundles (but are not yet aggregated), the light-grey matrix comprising the rest of polymer material is not yet expanded; $\Delta$ is the typical gap between the grey domains before their collapse. A region of the matrix shown in light-green is undeformed on the left, and is strongly stretched in the radial direction on the right. (b) The same structure in the xz plane: before the deformation of the network (top); after the deformation (bottom).

FIG. 7. Crosslinked polymer fiber of diameter $D_{f}$ surrounded by an annulus of pure solvent emerging during the late stage of phase separation. 

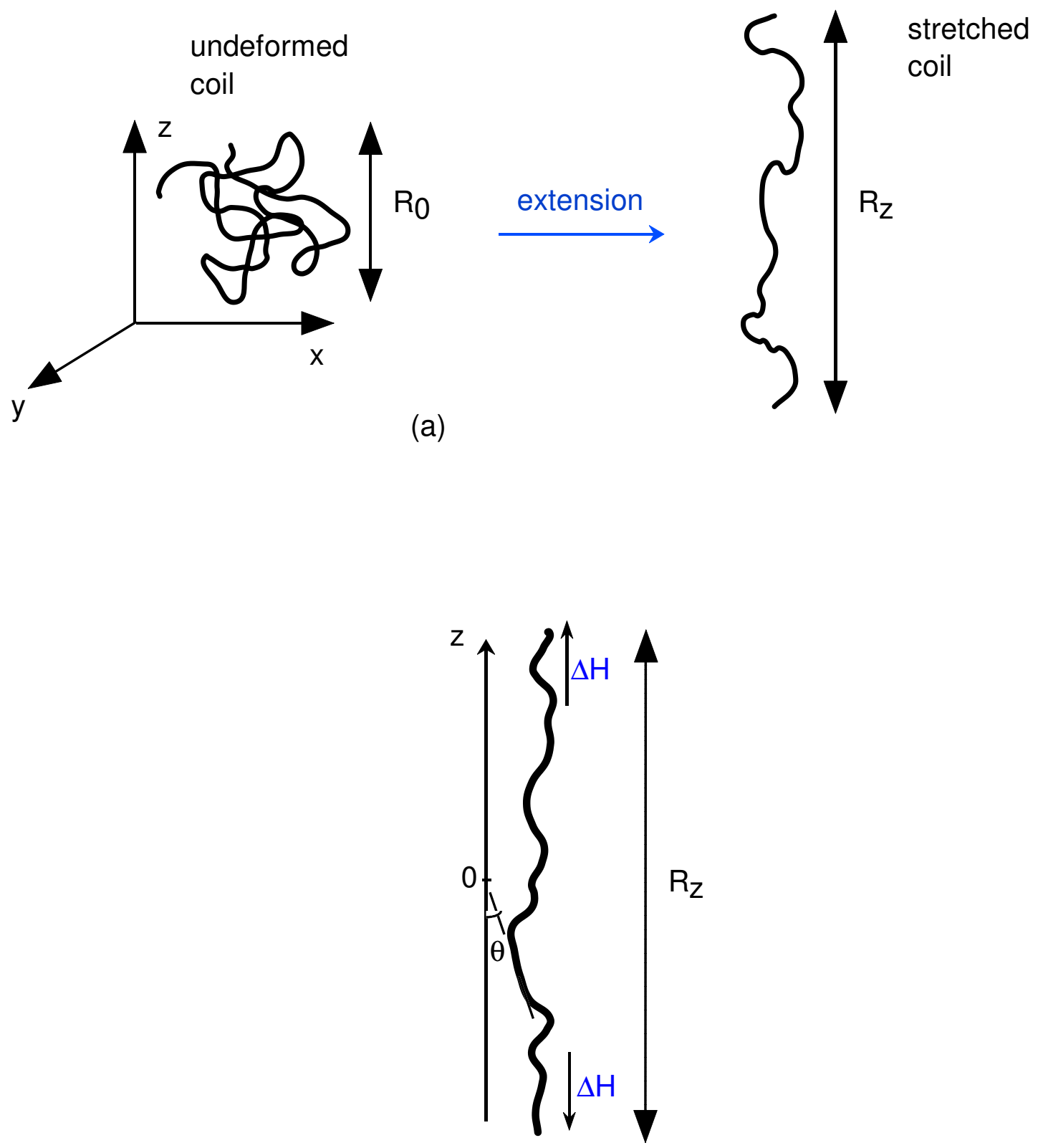

(b)

Fig. 1 


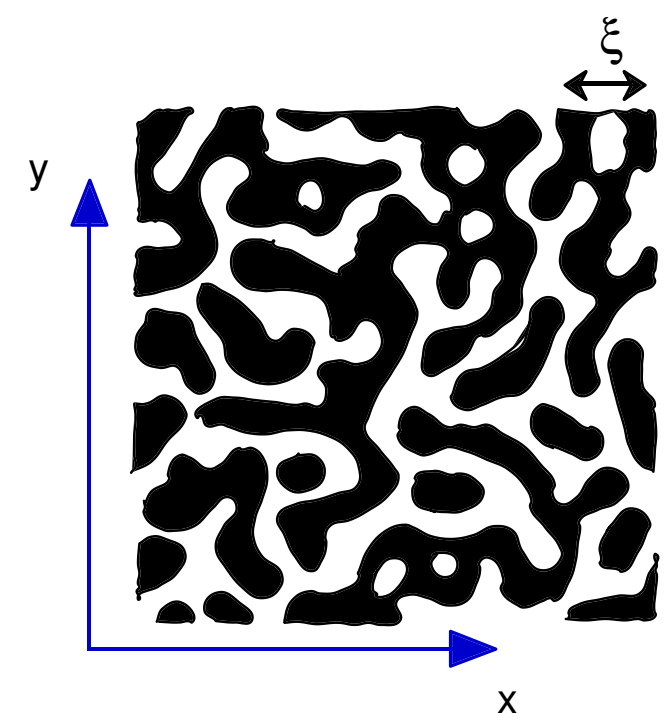

(a)

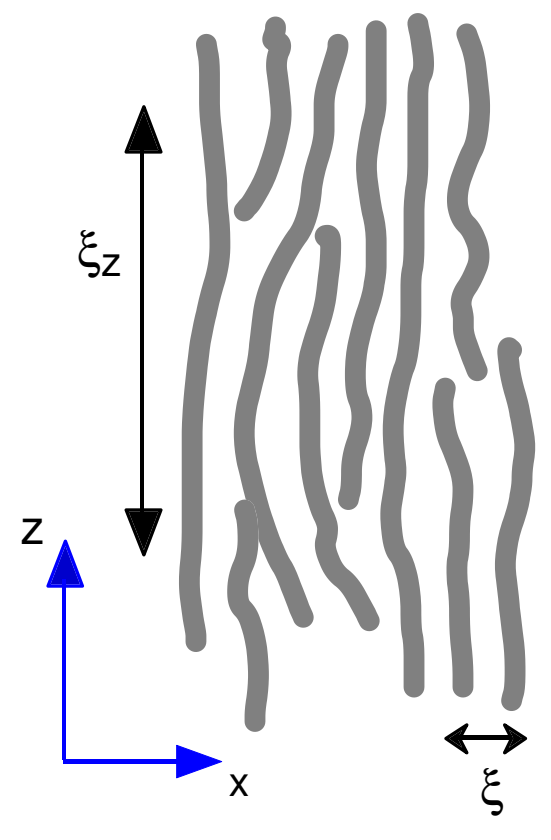

(b)

Fig. 2 

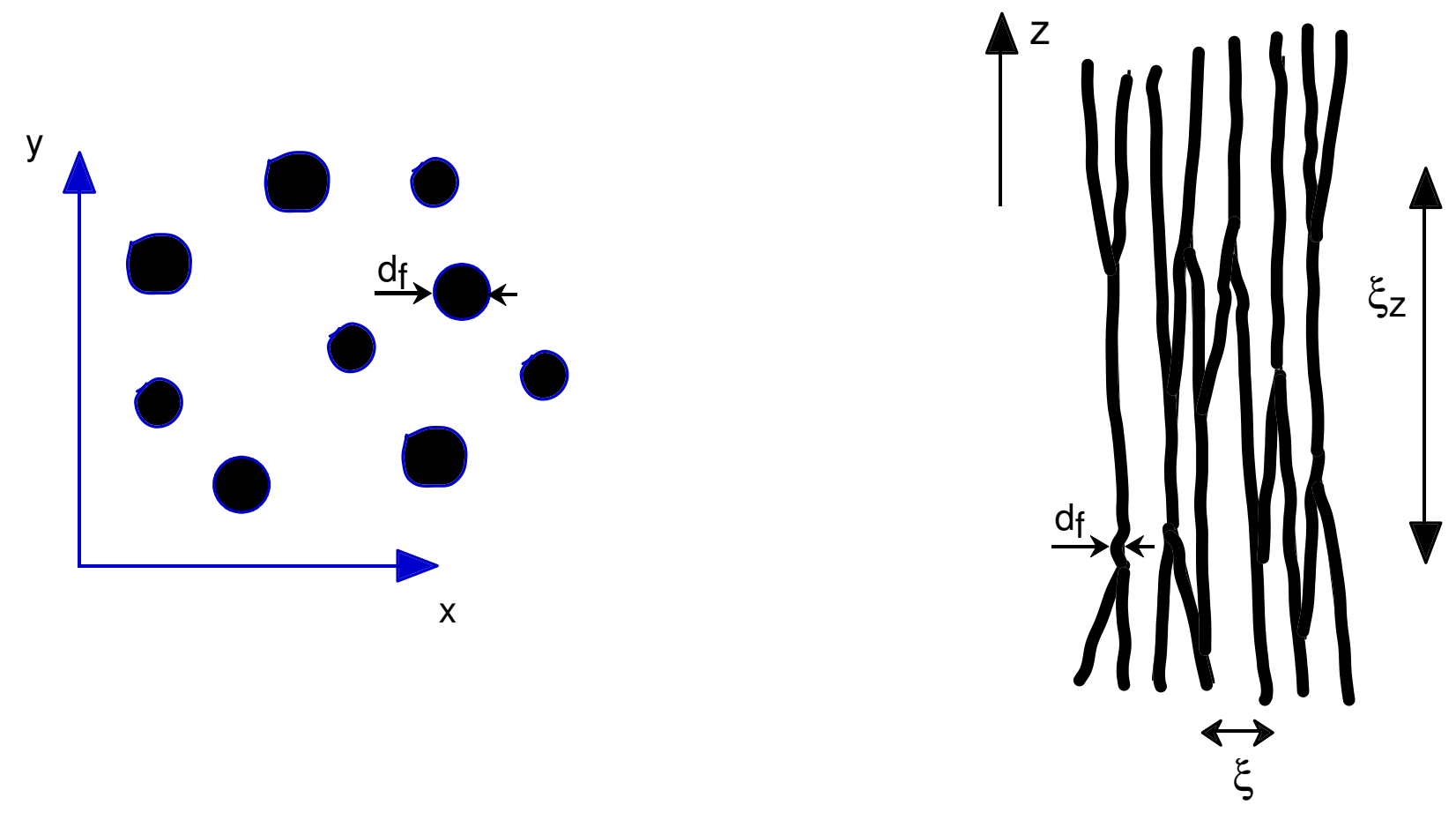

(a)

(b)

Fig. 3 


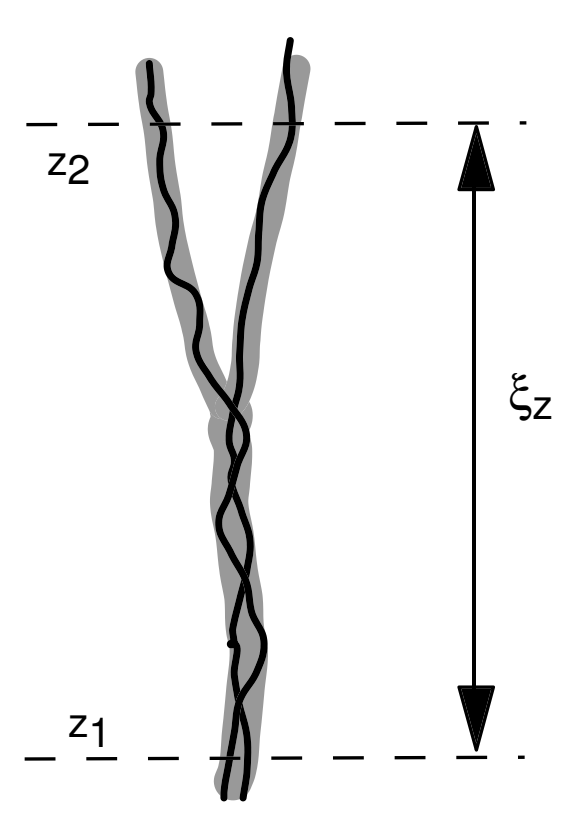

Fig. 4

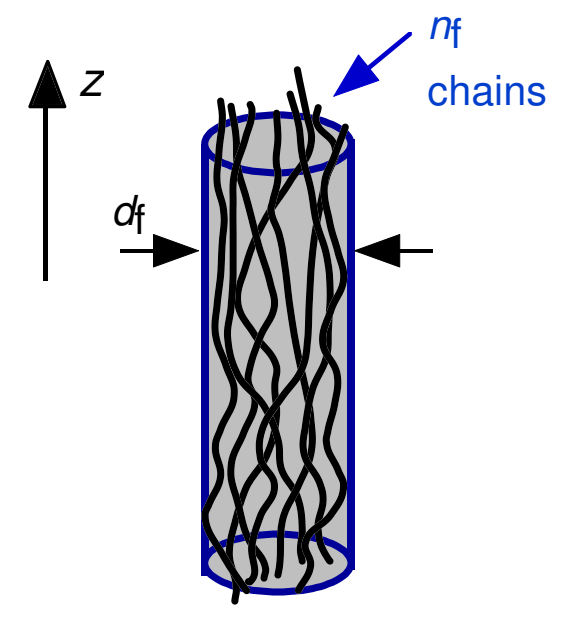

Fig. 5 

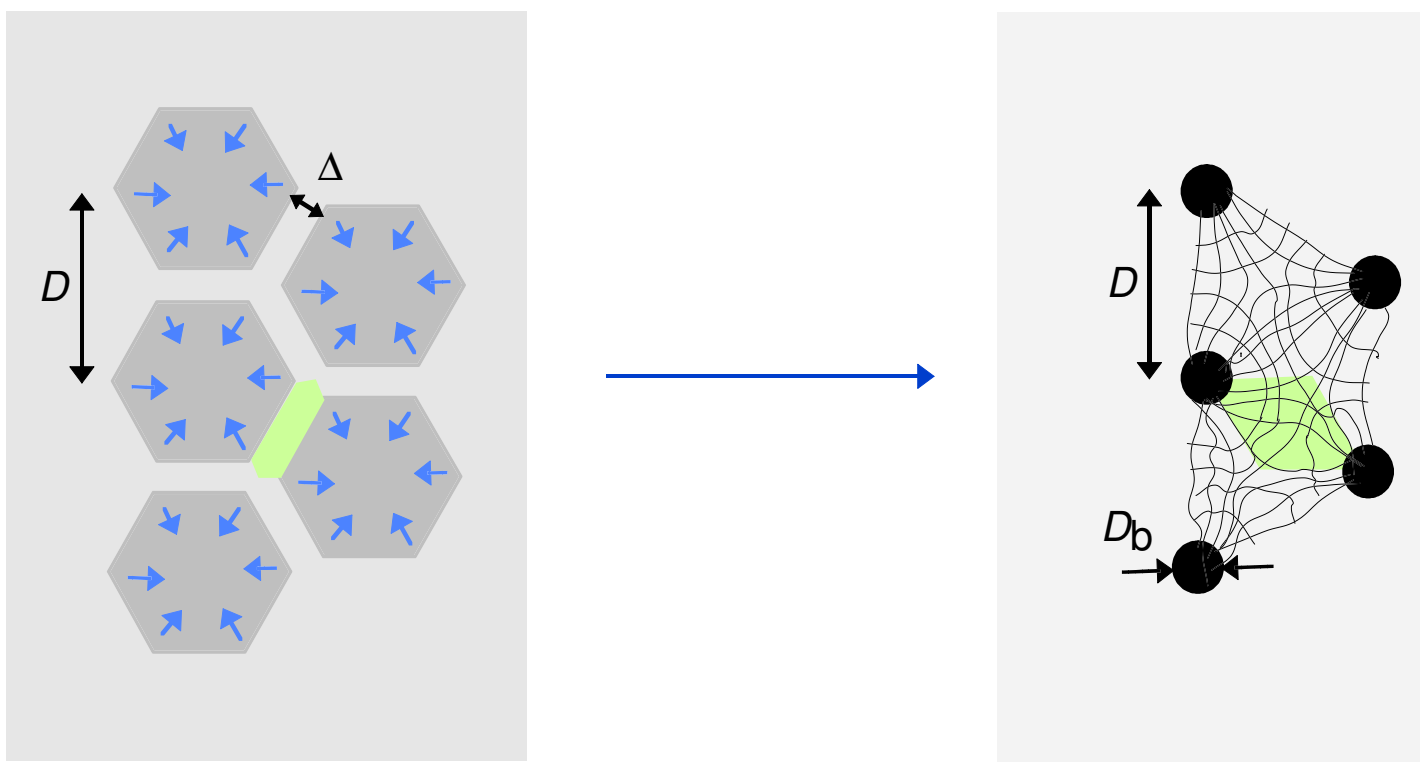

Fig. $6 a$

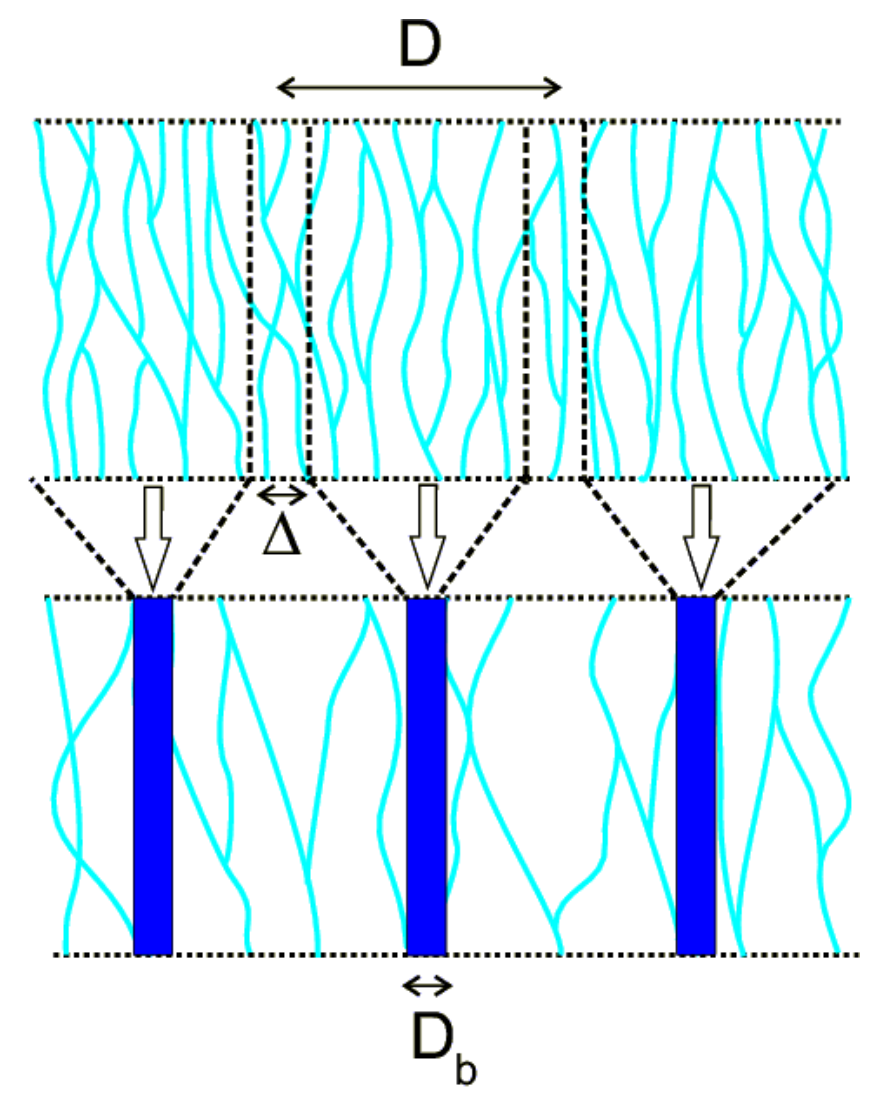

Fig. 6b 


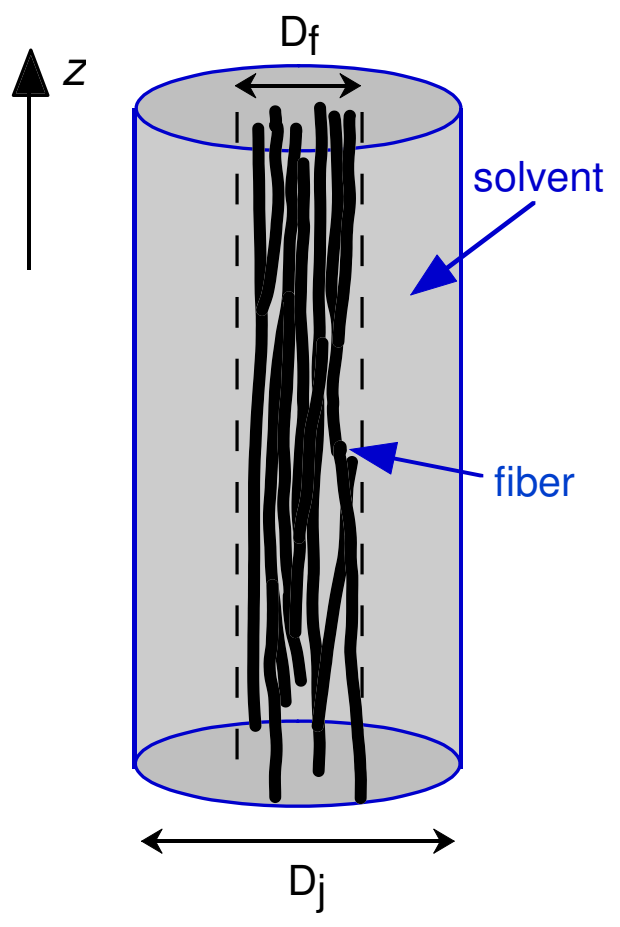

Fig. 7 